\title{
PHOTONICS Research
}

\section{Modeling of a SiGeSn quantum well laser}

\author{
Bahareh Marzban, ${ }^{1,2}$ Daniela Stange, ${ }^{2,3}$ Denis Rainko, ${ }^{2,3}$ Zoran Ikonic, ${ }^{4}$ Dan Buca, ${ }^{2,3}$ and \\ JeREMY WITZENS ${ }^{1,2, *}$ (1) \\ ${ }^{1}$ Institute of Integrated Photonics, RWTH Aachen University, 52074 Aachen, Germany \\ 2 Jülich-Aachen Research Alliance (JARA), Fundamentals of Future Information Technologies, Germany \\ ${ }^{3}$ Peter Grünberg Institute, 52428 Jülich, Germany \\ ${ }^{4}$ University of Leeds, School of Electronic and Electrical Engineering, Woodhouse, Leeds LS2 9JT, UK \\ ${ }^{*}$ Corresponding author: jwitzens@iph.rwth-aachen.de
}

Received 2 December 2020; revised 25 February 2021; accepted 30 March 2021; posted 31 March 2021 (Doc. ID 416505); published 15 June 2021

\begin{abstract}
We present comprehensive modeling of a SiGeSn multi-quantum well laser that has been previously experimentally shown to feature an order of magnitude reduction in the optical pump threshold compared to bulk lasers. We combine experimental material data obtained over the last few years with $k \cdot p$ theory to adapt transport, optical gain, and optical loss models to this material system (drift-diffusion, thermionic emission, gain calculations, free carrier absorption, and intervalence band absorption). Good consistency is obtained with experimental data, and the main mechanisms limiting the laser performance are discussed. In particular, modeling results indicate a low non-radiative lifetime, in the 100 ps range for the investigated material stack, and lower than expected $\Gamma$-L energy separation and/or carrier confinement to play a dominant role in the device properties. Moreover, they further indicate that this laser emits in transverse magnetic polarization at higher temperatures due to lower intervalence band absorption losses. To the best of our knowledge, this is the first comprehensive modeling of experimentally realized SiGeSn lasers, taking the wealth of experimental material data accumulated over the past years into account. The methods described in this paper pave the way to predictive modeling of new (Si) GeSn laser device concepts. () 2021 Chinese Laser Press
\end{abstract}

https://doi.org/10.1364/PRJ.416505

\section{INTRODUCTION}

Despite the fast progress made in silicon photonics (SiP), with silicon $(\mathrm{Si})$ waveguide-based devices [1], electro-optic modulators [2], and germanium $(\mathrm{Ge})$ photodetectors [3] made by complementary metal oxide semiconductor (CMOS) compatible fabrication, direct epitaxial integration of an efficient light source on $\mathrm{Si}$ remains a challenging goal. Commercialized SiP products rely on either hybrid or heterogeneous integration of III-V lasers, wherein a laser is packaged in either an optical micro-bench and subsequently attached to the chip [4] or an indium phosphide (InP) gain material wafer-bonded and subsequently processed at the wafer scale [5]. Direct growth of III-V gain materials on a Si substrate is challenging due to substantial lattice mismatch as well as the formation of anti-phase domains. Growth of electrically pumped III-V lasers on off-cut or V-groove templated Si has recently undergone substantial progress [6,7]. However, thick buffers make direct evanescent coupling to the underlying Si layer challenging. While bufferless III-V lasers have been grown on silicon using a combination of $\mathrm{V}$-grooved substrates and aspect ratio trapping of defects [8], these lasers have yet to be electrically pumped and experimentally coupled to Si waveguides [9]. While there also exists an interest to integrate III-V materials with CMOS for highperformance electronics applications [10], the introduction of III-V materials into front-end processes remains very problematic for currently accessible fabrication facilities.

The realization of an efficient, room temperature, and electrically pumped laser based on group IV materials would thus have several advantages. There have been numerous studies towards this objective, in which Ge continues to play a prominent role. Although Ge is an indirect bandgap material, its conduction band energy at the $\Gamma$-point is only $\sim 140 \mathrm{meV}$ above its indirect conduction band minimum at the L-point. Room temperature lasing has been achieved combining tensile strain and heavy n-doping [11], but with high optical pump lasing thresholds of $\sim 30 \mathrm{~kW} / \mathrm{cm}^{2}$ (corresponding to $\sim 35 \mathrm{kA} / \mathrm{cm}^{2}$ equivalent electrical current density). Electrically pumped lasers [12] have been reported at even larger thresholds of $280 \mathrm{kA} / \mathrm{cm}^{2}$, which resulted in very limited device lifetimes [13] and have proven difficult to reproduce or to improve upon.

More recently, Ge-tin (GeSn) [14] has emerged as a promising material for direct integration of group IV semiconductor lasers onto the SiP platform. While its reduced bandgap results in emission in the longer wavelength IR-B infrared range, at 
which $\mathrm{SiO}_{2}$ becomes increasingly absorptive [15], thus precluding the use of standard glass fiber for long distance communications, its potential for short-scale interconnects as well as sensing in the IR-B to mid-infrared (MIR) wavelength range was recognized early on $[16,17]$.

Incorporating $\mathrm{Sn}$ atoms into the Ge lattice modifies its electronic band structure. The energy of the direct transition at the $\Gamma$-valley reduces faster than the indirect transition from the L-valley, leading to a cross-over to a fundamental direct bandgap semiconductor once a critical amount of $\mathrm{Sn}$, estimated at about $\sim 8$ at. $\%$ (atomic fraction) in unstrained materials, is reached. Shortly after the first experimental indication of a GeSn alloy with a fundamental direct bandgap in 2014 [18], the first optically pumped GeSn laser was demonstrated at low cryogenic temperatures in 2015 [19]. Since then, different $\mathrm{GeSn}$-based structures aiming at reducing lasing thresholds and increasing operating temperatures have been investigated. Using $\mathrm{GeSn}$ bulk and $\mathrm{GeSn} / \mathrm{SiGeSn}$ double heterostructure (DHS) gain media, the maximum lasing temperature has been progressively increased from $110-130 \mathrm{~K}[20,21]$, to $180 \mathrm{~K}$ [22], $230 \mathrm{~K}$ [23], and $270 \mathrm{~K}[24,25]$. This trend has been driven mainly by improvements in growth methods, allowing higher $\mathrm{Sn}$ incorporation and increasing the energy separation between the $\Gamma$ - and L-valleys. Near room temperature lasing has been achieved for GeSn alloys with 20 at. \% Sn content [24] or 16 at. \% Sn under high uniaxial tensile strain [25]. However, optical pumping thresholds remained high. This is at least partly attributed to strong non-radiative recombination due to crystal defects in these metastable materials.

Historically, about an order of magnitude reduction in lasing threshold was achieved for III-V lasers every time the dimensionality of the gain material was reduced [26]. First attempts to follow this path with GeSn were hindered by the availability of suitable barrier materials. It was shown that using Ge barriers resulted in carrier leakage from quantum wells (QWs) due to inadequate energy barriers for holes [27]. A first step was achieved in 2017 with efficient electro-luminescence in GeSn multi-QWs (MQWs) [28] followed by demonstration of the first GeSn MQW laser in 2018 [29], both works using $\mathrm{SiGeSn}$ as a barrier material. It allowed reducing the optical threshold to $35 \mathrm{~kW} / \mathrm{cm}^{2}$ at cryogenic temperatures, about an order of magnitude lower compared to earlier experiments with bulk materials. The maximum lasing temperature, $120 \mathrm{~K}$ when using a $1550 \mathrm{~nm}$ pump laser, remained low, constrained not only by the relatively small directness of the utilized 13.3 at. \% Sn gain material, but also by finite barrier heights.

Using ternary SiGeSn alloys as barrier materials offers more degrees of freedom in adjusting the QW depth, while at the same time balancing lattice strain and mismatch. However, this poses further challenges related to epitaxial growth when using chemical vapor deposition (CVD) as typically used for Si CMOS technology and used so far in successful GeSn laser demonstrations [19-25,29]. Although a higher Sn content in the $\mathrm{GeSn}$ active region increases the maximum lasing temperature, its low growth temperature limits the achievable $\mathrm{Si}$ content in SiGeSn barrier layers due to limited precursor cracking [30,31]. However, a high Si content is required to increase the bandgap of the SiGeSn barrier material and achieve sufficiently good carrier confinement.

A second breakthrough in regard to threshold reduction has been recently achieved by utilizing a highly biaxially tensile strained, low 5.4 at. \% Sn content material. A low threshold of $1.1 \mathrm{~kW} / \mathrm{cm}^{2}$ was achieved together with lasing in continuous wave $(\mathrm{CW})$ operation [32]. This very significant improvement is at least partially attributed to the improved material quality, and thus reduced non-radiative recombination, at this low Sn content grown at higher temperatures. However, the maximum lasing temperatures have here also been limited to $100 \mathrm{~K}$, as a consequence of the low $\mathrm{Sn}$ content and the resulting low directness of the material.

Very recently, an electrically pumped GeSn device has been shown to lase in pulsed operation up to $100 \mathrm{~K}$ with a maximum quantum efficiency of $0.3 \%$ [33].

With the demonstration of low optical pump thresholds, $\mathrm{CW}$ operation, near room temperature lasing, electrical pumping, and concepts for waveguide coupling [34], GeSn continues to exhibit a high potential for the realization of practically relevant group IV lasers. Outstanding challenges are not only to achieve CW electrical pumping, but also to combine a low lasing threshold and increased temperature lasing, as well as to reach reasonable wall-plug efficiencies. In view of this, improving the accuracy of device modeling based on the experimental work done over the last years is of paramount importance.

Early efforts to model DHS and MQW lasers $[35,36]$ pointed out the benefits of using $\mathrm{SiGeSn}$ as a cladding material, as well as the potential of MQWs to reduce lasing thresholds and suppress Auger recombination. However, these studies were lacking the wealth of experimental data that has since been accumulated and have been done on simplified device geometries. Here, we have chosen to model in detail the MQW laser previously published by Stange et al. [29], to verify and refine model assumptions in view of developing the predictive modeling tools required to identify the best structures for practical GeSn lasers.

Temperature, mode, and pump intensity dependent net gain is modeled to predict experimentally determined lasing thresholds. This provides insights into the main mechanisms limiting the efficiency and temperature requirements of the device, as well as guidelines on how to further improve its characteristics. To calculate the gain, carrier concentrations are first determined by modeling optical absorption and applying the drift-diffusion, Poisson, and thermionic emission models using semiconductor material parameters obtained from $k \cdot p$ theory. From the carrier concentrations, optical gain as well as free carrier absorption (FCA), including intervalence band absorption (IVBA), is calculated. Beyond pointing out the main factors limiting the performance of the device, this modeling also provides additional insights into carrier lifetimes as well as energy band bowing and band alignment parameters. As the models developed here continue to be applied to further experimental results, they will continue to gain in accuracy.

Section 2 describes the modeled device. Sections 3 and 4 describe modeling assumptions regarding carrier transport and gain/loss calculations, respectively, and may be skipped by a reader more interested in the modeling results described 
Table 1. Summary of Assumed Material Properties in the 50-100 K Range

\begin{tabular}{|c|c|c|c|}
\hline & $\begin{array}{l}\mathrm{Ge}_{0.867} \mathrm{Sn}_{0.133} \\
\text { (gain medium) }\end{array}$ & $\begin{array}{c}\mathrm{Si}_{0.048} \mathrm{Ge}_{0.822} \mathrm{Sn}_{0.130} \\
\text { (barrier layers) }\end{array}$ & $\begin{array}{c}\mathrm{Ge}_{0.9} \mathrm{Sn}_{0.1} \\
\text { (buffer layer) }\end{array}$ \\
\hline$\overline{\Gamma \text {-valley mobility at } 5 \times 10^{17} \mathrm{~cm}^{-3} \text { total carrier concentration }}$ & $3500 \mathrm{~cm}^{2} /(\mathrm{V} \cdot \mathrm{s})$ & $1850 \mathrm{~cm}^{2} /(\mathrm{V} \cdot \mathrm{s})$ & $3190 \mathrm{~cm}^{2} /(\mathrm{V} \cdot \mathrm{s})$ \\
\hline Average hole mobility at $5 \times 10^{17} \mathrm{~cm}^{-3}$ total carrier concentration & $500 \mathrm{~cm}^{2} /(\mathrm{V} \cdot \mathrm{s})$ & $500 \mathrm{~cm}^{2} /(\mathrm{V} \cdot \mathrm{s})$ & $500 \mathrm{~cm}^{2} /(\mathrm{V} \cdot \mathrm{s})$ \\
\hline Carrier lifetime (final model) & 80 ps & 80 ps & 80 ps \\
\hline Background p-type doping & $2 \times 10^{17} \mathrm{~cm}^{-3}$ & $2 \times 10^{17} \mathrm{~cm}^{-3}$ & $2 \times 10^{17} \mathrm{~cm}^{-3}$ \\
\hline Refractive index at $2.5 \mu \mathrm{m}$ & 4.2 & 4.2 & 4.1 \\
\hline \multirow[t]{3}{*}{ Luttinger parameter } & $\gamma_{1}=19.29$ & $\gamma_{1}=18.96$ & $\gamma_{1}=16.21$ \\
\hline & $\gamma_{2}=7.36$ & $\gamma_{2}=7.21$ & $\gamma_{2}=5.84$ \\
\hline & $\gamma_{3}=8.80$ & $\gamma_{3}=8.64$ & $\gamma_{3}=7.26$ \\
\hline Top surface recombination velocity & & $10,000 \mathrm{~cm} / \mathrm{s}$ & \\
\hline Bottom surface recombination velocity & & $\infty$ & \\
\hline
\end{tabular}

Table 2. Summary of Gain Modeling Assumptions

\begin{tabular}{lc}
\hline Parameter & Value \\
\hline Gain broadening & $25 \mathrm{meV}$ \\
Bandgap narrowing at $n=2 \times 10^{17} \mathrm{~cm}^{-3}$ & $24 \mathrm{meV}$ \\
Bandgap narrowing at $n=2 \times 10^{18} \mathrm{~cm}^{-3}$ & $37 \mathrm{meV}$ \\
Correction to Drude model absorption, electrons & $2 \times$ \\
Correction to Drude model absorption, holes & $2 \times$ \\
Roughness correlation length $\left(L_{c}\right)$ & $500 \mathrm{~nm}$ \\
Roughness amplitude $(\sigma)$ & $55 \mathrm{~nm}$ \\
\hline
\end{tabular}

in Section 5 (a summary of modeling assumptions can be found in Tables 1 and 2 at the ends of Sections 3 and 4). A discussion of the modeling results, an outlook, and conclusions are given in Section 6.

\section{DEVICE DESCRIPTION}

We focus on the structure referred to as MQW-A in Ref. [29], which features better properties in regard to lasing, as evidenced, for example, by its comparatively low lasing thresholds at cryogenic temperatures. The gain material consists of $1022 \mathrm{~nm} \mathrm{Ge} e_{0.867} \mathrm{Sn}_{0.133}$ QWs separated by $22 \mathrm{~nm}$ $\mathrm{Si}_{0.048} \mathrm{Ge}_{0.822} \mathrm{Sn}_{0.130}$ barrier layers. The stack is capped with a $50 \mathrm{~nm} \mathrm{Si}{ }_{0.048} \mathrm{Ge}_{0.822} \mathrm{Sn}_{0.130}$ layer and grown on a $200 \mathrm{~nm}$ thick $\mathrm{Ge}_{0.90} \mathrm{Sn}_{0.10}$ buffer layer, as shown in Fig. 1(a). The whole stack was grown on a $2.5 \mu \mathrm{m}$ thick Ge virtual substrate (VS). Due to the close match between the lattice constant of the buffer and the average lattice constant of the remaining layer stack, the whole $(\mathrm{Si}) \mathrm{GeSn}$ stack could be grown free of internal misfit dislocations, which remain confined to the interface between the Ge-VS and the $\mathrm{Ge}_{0.90} \mathrm{Sn}_{0.10}$ buffer layer. An $8 \mu \mathrm{m}$ diameter microdisk [38] was etched into the stack and the Ge-VS underetched and reduced to a $0.8 \mu \mathrm{m}$ wide Ge pillar. This allows the stress accumulated during growth to relax to a large extent, with residual strain due mostly to mismatch between the layers. This substantially improves the electronic band structure characteristics of the structure, since the compressive strain present in the as-grown material reduces the directness of the GeSn QW material [29].
Strain engineering in suspended microdisk lasers has resulted in the lowest thresholds demonstrated to date [32]; however, it also comes with a number of challenges related to contacting and heat sinking that first need to be addressed to realize electrically pumped structures. Similar challenges have, for example, been faced in electrically pumped photonic crystal suspended membrane lasers [39] in which a pedestal supporting the cavity was used to conduct both current and heat to the chip's substrate. We are currently working on an electrically pumped suspended GeSn microdisk laser concept, a description of which can be found in Ref. [40].

The strain across the undercut layer stack, along a vertical axis close to the periphery of the disk, where whispering gallery modes are confined and the optical field has its maximum, is shown in Fig. 1(b). The top QW is an almost fully relaxed layer with a $-0.05 \%$ residual compressive strain, while the bottom QW still experiences a $-0.2 \%$ residual strain as a consequence of the bimetal-like deformation of the disk [29]. While an inhomogeneous strain distribution across the device is generally undesirable, as it leads to different optical transition energies across the different QWs, the spread is sufficiently small here for all QWs to efficiently contribute gain to the lasing mode [29].

While modeling carrier concentrations as well as material gain and losses is done only along the axis shown in Fig. 1(a), this is sufficient to fully account for material properties across the optical mode profiles. As shown with mechanical modeling in the Supplementary Information in Ref. [29], for the thin pedestal geometry of this device, the strain is homogeneous across the $(x y)$ plane of the disk other than in a small region at its very center immediately above the pedestal (as opposed to what happens with thicker pedestals, as shown in the Supplementary Information in Ref. [20]). Given the 80 ps lifetimes considered in the following and the highest carrier mobilities of $3500 \mathrm{~cm}^{2} /(\mathrm{V} \cdot \mathrm{s})$ associated with $\Gamma$-valley electrons, diffusion lengths at $100 \mathrm{~K}$ are below $500 \mathrm{~nm}$, so that the surface recombination at the periphery of the disk also ceases to affect carrier concentrations for points deeper inside the disk. Moreover, even assuming a very high surface recombination velocity of $10,000 \mathrm{~cm} / \mathrm{s}$ (see Section 3), carrier lifetimes are 
(a)

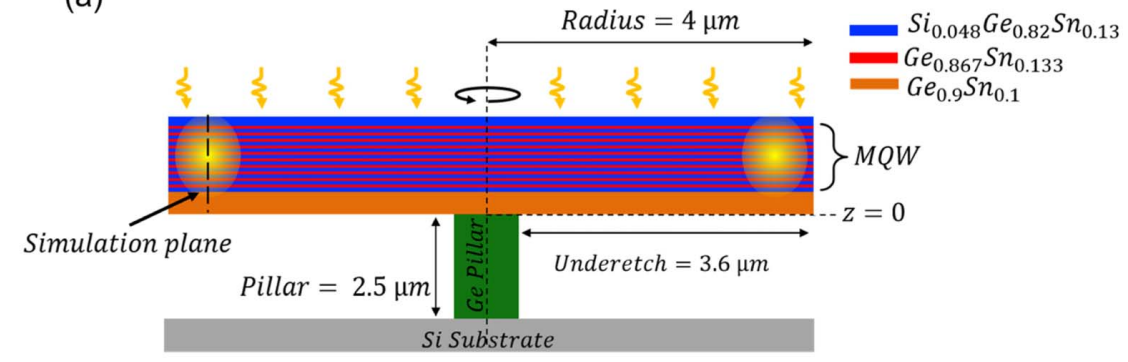

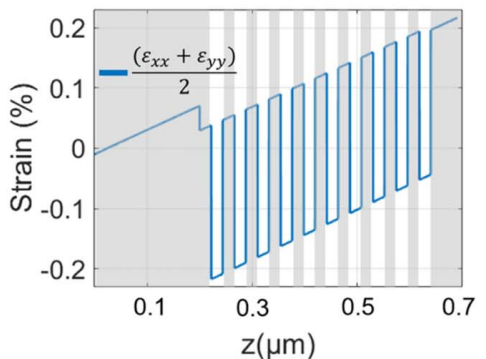

(b)

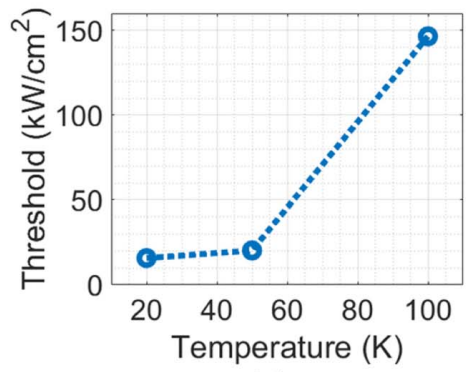

(c)

Fig. 1. Modeled microdisk laser. (a) Schematic cross-section of the disk with labeled dimensions. (b) Strain across the layer stack, along a vertical axis near the periphery of the disk, where the optical field of whispering gallery ground modes is maximal. (c) Measured thresholds reported by Stange et al. [29] at a $1064 \mathrm{~nm}$ pump wavelength (Nd:YAG).

limited only by surface recombination in the first $\sim 50 \mathrm{~nm}$ from the surface, after which bulk Shockley-Read-Hall $(\mathrm{SRH})$ recombination becomes the limiting factor. Since this is much smaller than the radial extent of the relevant optical modes, surface recombination at the outer disk periphery does not play a significant role in the modal properties given the low bulk recombination rates considered in the following.

As part of the experimental characterization of this device, temperature dependent optical pump thresholds were determined for both $1550 \mathrm{~nm}$ and $1064 \mathrm{~nm}$ pump lasers. A more detailed analysis of the optical excitation, together with the exact mode profiles, has led to a downward correction of the initially reported excitation thresholds. In Ref. [29], the optical intensity was calculated by dividing the total beam power by the area of a disk whose diameter coincides with the full width at half maximum (FWHM) of the beam. Here, we rather take the exact Gaussian shape of the beam, as reported in the Supplementary Information in Ref. [29], at the location of the whispering gallery modes into account. The updated thresholds for a $1064 \mathrm{~nm}$ pump are shown in Fig. 1(c). It is apparent that a relatively low threshold, $16 \mathrm{~kW} / \mathrm{cm}^{2}$, is obtained at $20 \mathrm{~K}$ that rapidly increases between $50 \mathrm{~K}$ and $100 \mathrm{~K}$, from $20 \mathrm{~kW} / \mathrm{cm}^{2}$ to $146 \mathrm{~kW} / \mathrm{cm}^{2}$. Laser action ceases shortly thereafter as the temperature is further increased (100 K was the maximum lasing temperature for the $1064 \mathrm{~nm}$ pump).

\section{TRANSPORT ASSUMPTIONS AND MODELS}

The carrier concentrations are modeled with Synopsys' Sentaurus Device (sDevice) modeling tool. This section gives a detailed justification for the modeling assumptions summarized in Table 1.
The Nd:YAG pump laser emits $5 \mathrm{~ns}$ pulses at a $1064 \mathrm{~nm}$ center wavelength and with a small duty cycle of $8.5 \times 10^{-5}$. The non-radiative carrier lifetime has been measured to be much shorter, in the one or few 100 ps range for GeSn layers of comparable Sn content and grown in the same CVD chamber $[41,42]$, as discussed in more detail below. Moreover, the photon lifetime is also expected to be significantly below 100 ps, which would correspond to a high resonator quality factor of 75,000 . Thus, the carrier dynamics can be safely assumed to reach steady-state conditions and to adiabatically track the instantaneous pulse power. The temperature increase during the pulse duration is evaluated based on the deposited energy and the specific heat of the material. Given the short pulses, we conservatively neglect thermal conductivity during their duration. While the thermal conductivity of GeSn has been intensely investigated [43], much fewer reports are available on its specific heat, so we assume here the temperature dependent specific heat of Ge [44]. We estimate the temperature increase during the pulse duration to be less than $10 \mathrm{~K}$ at the $146 \mathrm{~kW} / \mathrm{cm}^{2}$ threshold at $100 \mathrm{~K}$. These temperature cycles have a relatively small magnitude, so their dynamics are not considered further in the simulations. Thus, steadystate simulations are run assuming the peak pulse power shown in Fig. 1(c) [29].

As the first step, carrier generation resulting from optical pumping is computed. The optical generation rate is calculated as $G_{\mathrm{opt}}=\alpha I(d) / \hbar \omega$, where $I(d)$ is the light intensity at depth $d$, determined via the transfer matrix method (TMM), and $\hbar \omega$ is the photon energy. The absorption coefficients at the $1064 \mathrm{~nm}$ wavelength are extracted from ellipsometry measurements (made on thick, partially strain-relaxed layers) as $\alpha=5 \times 10^{4}-6 \times 10^{4} \mathrm{~cm}^{-1}$ in the QW and barrier layers and 
as $4 \times 10^{4} \mathrm{~cm}^{-1}$ in the $\mathrm{Ge}_{0.90} \mathrm{Sn}_{0.10}$ buffer layer. The refractive index is extracted as $n \simeq 4.5$, well in line with literature values [45]. At the laser emission wavelength, close to $2.5 \mu \mathrm{m}$, the refractive index was extracted as $\sim 4.2$ in the MQW stack and $\sim 4.1$ in the buffer.

Strain and material composition dependent energy levels and anisotropic effective masses were obtained from eight-band $k \cdot p$ theory [46] for the $\Gamma$ and $\mathrm{L}$ conduction band valleys as well as for the heavy hole (HH) and light hole (LH) bands, with the band-energy bowing parameters used in Ref. [37]. Concentration dependent Luttinger parameters were interpolated from the data reported by Low et al. [47]. Band alignments at heterojunctions were obtained based on alloy dependent average valence band energies of the bulk materials obtained following Ref. [48], as further explained in Ref. [37].

Spatially dependent optical carrier generation creates a net diffusion current from the top surface of the disk towards the substrate. This carrier transport is modeled self-consistently with the Poisson equation, as well as with drift-diffusion inside the bulk of the layers. At heterostructure boundaries, currents are modeled with thermionic emission. Quantization within the QWs and modification of the local density of states (LDOS) are handled with the QWLocal model within sDevice [49], since it is compatible with the definition of multiple valence and conduction band valleys, which is an essential feature for the modeling of $\mathrm{GeSn}$-based devices due to the small energy differences. Since sDevice assumes electron and hole concentrations are locally at thermo-dynamic equilibrium relative to their respective quasi-Fermi levels, hot carrier distributions not locally defined by Fermi-Dirac statistics are ignored.

The $20 \mathrm{~K}$ flat-band energy diagrams, before applying optical pumping, of both the conduction and valence bands can be seen in Figs. 2(a) and 2(b). Even though strain is not strictly biaxial due to the deformation of the disk [29], holes are classified as $\mathrm{HH}$ or $\mathrm{LH}$ depending on whether they present higher or smaller mass $m_{z}$ in the direction of quantization, referred to as the $z$ direction.

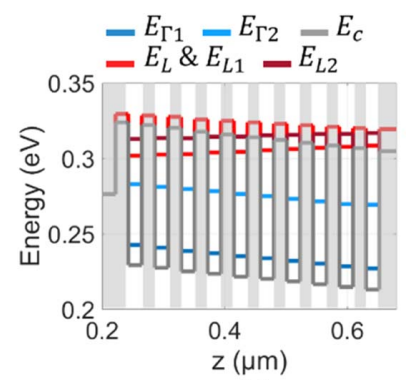

(a)

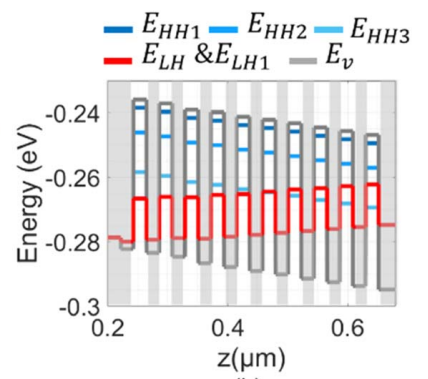

(b)
Fig. 2. Flat-band energy diagram at $20 \mathrm{~K}$. (a) Conduction band energy levels, with quantization levels in the wells (the gray curve shows the non-quantized $E_{\Gamma}$ band edge energy, the blue curves the quantized $E_{\Gamma 1}$ and $E_{\Gamma 2}$ levels, and the red curve $E_{\mathrm{L} 1}$ inside the wells and the nonquantized $E_{\mathrm{L}}$ inside the barriers). (b) Valence band energy levels, with quantization levels in the wells (the gray curve shows the nonquantized $E_{\mathrm{HH}}$ band edge, the blue curves the $E_{\mathrm{HH} 1}, E_{\mathrm{HH} 2}$, and $E_{\mathrm{HH} 3}$ levels, and the red curve $E_{\mathrm{LH} 1}$ inside the wells and the nonquantized $E_{\mathrm{LH}}$ inside the barriers).
Electrons see a QW depth of $\sim 93 \mathrm{meV}$, as determined by the $\Gamma$-valley energies before quantization seen in Fig. 2(a). Holes see much smaller QW depths varying between $44 \mathrm{meV}$ and $28 \mathrm{meV}$ as one moves from the lowest (No. 1) to the highest (No. 10) QW in the structure [Fig. 2(b)], as a consequence of the reduced compressive strain in the gain material and simultaneously increased tensile strain in the barrier material affecting the LH-HH energy splitting [Fig. 1(b)]. The QW depths are determined by the HH energy in the QWs and the LH energy in the barriers that shift in different directions as the strain is varied.

Figure 2 also shows the quantized energy levels for all carrier types. There are two quantized $\Gamma$-valley electron levels $E_{\Gamma 1}$ and $E_{\Gamma 2}$ in each $\mathrm{QW}$, as well as two L-valley levels $E_{\mathrm{L} 1}$ and $E_{\mathrm{L} 2}$ (three after reduction of the $\Delta E_{\mathrm{L}-\Gamma}$ energy difference in the Modeling Results section). In regard to the valence band, there are three quantized $\mathrm{HH}$ levels $E_{\mathrm{HH} 1}-E_{\mathrm{HH} 3}$ and a single $\mathrm{LH}$ level $E_{\mathrm{LH} 1}$.

The low energy difference between the $\mathrm{L}$ - and $\Gamma$-valleys, as well as the small splitting between HHs and LHs in strained layers, leads to population of multiple valleys that need to be taken into account. This is true even at very low temperatures, such as the $20 \mathrm{~K}$ at which the lowest temperature threshold has been measured [29], due to band filling. The energy difference between the $\mathrm{L}$ - and $\Gamma$-valleys $\left(\Delta E_{\mathrm{L}-\Gamma}\right)$ for the $\mathrm{Ge}_{0.867} \mathrm{Sn}_{0.133}$ gain material considered here, between $69 \mathrm{meV}$ and $92 \mathrm{meV}$ as one moves from the bottommost to the topmost QW, is substantially larger than $k_{B} T$ at $20 \mathrm{~K}(1.7 \mathrm{meV})$, with $k_{B}$ the Boltzmann constant and $T$ the temperature. However, the $\Gamma$-valley fills up rapidly due to its low effective electron mass $\left(0.025 m_{0}\right.$, with $m_{0}$ the free electron mass). The electron Fermi level reaches L-valley energies for concentrations as low as $5.7 \times 10^{17} \mathrm{~cm}^{-3}$ for the topmost QW $\left(2.9 \times 10^{17} \mathrm{~cm}^{-3}\right.$ after reducing $\Delta E_{\mathrm{L}-\Gamma}$ by $30 \mathrm{meV}$ in the Modeling Results section), in the same order as the low temperature threshold concentrations. Due to the even smaller energy difference between LHs and $\mathrm{HH}$ and the intrinsic p-type background doping (see below), LHs also play a role even at low temperature thresholds.

Carrier capture into and escape from the QWs are modeled with thermionic emission with coefficients adapted to the $\mathrm{SiGeSn}$ material, taking the effective masses and valley degeneracy of the SiGeSn barriers into account [50] and assuming negligible intervalley scattering during the thermionic emission process [51], as described in Appendix A.

Auger recombination is particularly strong in low bandgap semiconductors $[52,53]$, and is thus expected to play an important role in room temperature operation of $\mathrm{Ge}$ and $\mathrm{GeSn}$ lasers $[35,54]$. However, band-to-band Auger recombination is also highly temperature dependent [35,55], and drops rapidly at cryogenic temperatures. Given the Auger coefficients estimated for $\mathrm{GeSn}$ in the literature [35] and the high dark recombination rates attributed to SRH and surface recombination (due to insensitivity of the measured carrier lifetimes to carrier concentrations in Refs. $[41,42]$, we do not expect Auger recombination to play a dominant role in the $20 \mathrm{~K}$ to $120 \mathrm{~K}$ temperature range of the experimental results modeled here. In addition, QWs may further contribute to suppressing Auger recombination $[36,56]$. 
Carrier lifetimes previously measured in thin films grown in the same reactor provide guidance: carrier lifetimes of 435 ps and 170 ps were measured at $20 \mathrm{~K}$ and $25 \mathrm{~K}$, respectively, on non-undercut but partially relaxed $\mathrm{GeSn}$ films with a respective Sn content of 8.5 at. $\%$ and 12.5 at. \% and respective thicknesses of $800 \mathrm{~nm}$ and $560 \mathrm{~nm}$ [41]. At room temperature, these carrier lifetimes increased moderately, respectively, to 470 ps and 260 ps. Since both the alloy composition and the film thickness were varied, it could not be conclusively determined whether carrier lifetimes were limited by bulk or surface recombination, or a combination of the two. Indeed, both the dislocation network at the bottom of the films and bulk SRH recombination, possibly caused by the large density of vacancies typically observed in these materials [57] as a consequence of the low temperature growth process, could play a significant role in limiting the carrier lifetimes. In a more recent study [42], a carrier lifetime of 217 ps was measured in a relaxed, 12.5 at. \% Sn sample with a $350 \mathrm{~nm}$ thickness, relatively close to the previously reported 170 ps. Here too, it was concluded that lifetimes do not have a significant carrier concentration or temperature dependence. Since the carrier specific SRH minority lifetimes $\tau_{n}$ and $\tau_{p}$ are not individually known, we set them to be equal to each other and to half the assumed carrier lifetime. The exact partitioning between them is irrelevant here, as electron and hole concentrations remain close to each other in the following simulations. Since the main focus here is on modeling the pump levels required to reach lasing threshold, at which the high non-radiative recombination rates seen here are dominant, radiative recombination is not included in the models. Assumptions on carrier lifetimes and their consequences on modeling results are further discussed in the Modeling Results section.

We follow a worst-case approach for surface recombination velocities. It will be shown that relatively low bulk lifetimes have to be assumed to reproduce experimental results. These dominate dark recombination rates, such that even very high surface recombination rates do not appear to significantly impact the modeling results. The surface recombination velocity for bare Ge surfaces has been estimated to be above $1000 \mathrm{~cm} / \mathrm{s}$ [58]. Although the microdisk modeled here has been passivated with a $10 \mathrm{~nm}$ layer of $\mathrm{Al}_{2} \mathrm{O}_{3}$ to reduce the surface trap density [59], the material properties of SiGeSn are largely unknown in this regard. Hence, we make a conservative assumption of $10,000 \mathrm{~cm} / \mathrm{s}$ for the top surface. This value is even above that of highly damaged, etched silicon surfaces, estimated at $4000 \mathrm{~cm} / \mathrm{s}$ [60].

The surface recombination velocity at the bottom surface, below the $\mathrm{Ge}_{0.90} \mathrm{Sn}_{0.10}$ buffer, is expected to be worse than at the top surface, since it corresponds to the interface where the misfit dislocation network is located. While the undercut might have removed part of these defects, its quality is expected to remain worse. We found the modeling results to be relatively insensitive to the precise recombination velocity assumption made, also as a consequence of the low carrier concentrations at the bottom surface. We model it as an ohmic contact, effectively setting the surface recombination velocity to infinity, forcing the electron concentration to zero and the hole concentration to the intrinsic background doping.
Significant p-type background doping has been observed to be present in nominally undoped $\mathrm{GeSn}$ films and to grow with Sn concentration as a result of the decreasing material quality. For a partially relaxed 12.5 at. \% Sn material close to the composition of the 13.3 at. \% gain material used here, hole concentrations have been independently shown to be on the order of $2 \times 10^{17} \mathrm{~cm}^{-3}$ by means of electrochemical capacitancevoltage (ECV) [61] and Hall measurements [62]. This level of p-doping is assumed throughout the $\mathrm{SiGeSn}$ layer stack in our simulations.

Previously reported Hall mobilities measured from samples fabricated in the same growth chamber serve as the basis for modeling in this work [62]. For holes, we take the experimental data recorded from partially relaxed 12.5 at. \% epitaxially grown GeSn films, with $\mu_{p} \simeq 700 \mathrm{~cm}^{2} / \mathrm{Vs}$ in the $50 \mathrm{~K}$ to $100 \mathrm{~K}$ range and intrinsic p-type concentrations in the $2 \times 10^{17} \mathrm{~cm}^{-3}$ to $3 \times 10^{17} \mathrm{~cm}^{-3}$ range. Since separate experimental mobility data for $\mathrm{HH}$ and $\mathrm{LHs}$ were not available for samples grown in this CVD chamber, we settled for these average experimental numbers. At cryogenic temperatures, the hole mobility showed very small temperature dependence and dropped to only $600 \mathrm{~cm}^{2} /(\mathrm{V} \cdot \mathrm{s})$ at $140 \mathrm{~K}$. Temperature and carrier concentration dependent mobilities of Ge [63] also feature low temperature sensitivity and a primary dependence on carrier concentration, resulting from carrier-carrier scattering, for concentrations above a few times $10^{17} \mathrm{~cm}^{-3}$.

For electrons, the modeling takes into account a number of corrections relative to the experimental data. On one hand, the $\Gamma$-valley mobility is very sensitive to the $\mathrm{Sn}$ content due to the decreasing effective mass as the $\mathrm{Sn}$ content is increased. On the other hand, the overall electron mobility is also very sensitive to temperature, strain, and the resulting ratio between $\Gamma$ - and L-valley electron concentrations, as $\Gamma$-valley mobility is much higher than L-valley mobility. To extract separate mobilities for $\Gamma$ - and L-valley electrons, we fit the experimental data [62] reported at different temperatures for 12.5 at. \% $\mathrm{GeSn}$, both for as-grown partially relaxed $(0.4 \%$ residual compressive strain) and fully relaxed undercut epitaxial films, taking into account the expected ratio between $\Gamma$ - and L-valley electrons based on Fermi-Dirac statistics. We then correct the extracted numbers for the slightly different conductivity effective masses for the different alloy compositions considered here, resulting in the estimates summarized in Table 1. Mobilities assumed in sDevice are then adjusted iteratively based on the modeled ratio of $\Gamma$ - to L-valley concentrations.

For both electrons and holes, the dependence of mobility on carrier concentrations is taken into account with the Arora model [64], as implemented in sDevice, with coefficients chosen to mimic the relative behavior of Ge mobilities.

A summary of assumed material properties can be found in Table 1. Tabulated effective masses and flat band energy levels are reported in the Supplementary Information in Ref. [65].

\section{ASSUMPTIONS AND MODELS FOR GAIN AND LOSS CALCULATIONS}

Carrier concentrations modeled in sDevice are used for evaluating gain and losses in the materials. Even though the majority of holes occupy the $\mathrm{HH}$ band, both the $\Gamma \rightarrow \mathrm{HH}$ and $\Gamma \rightarrow \mathrm{LH}$ 
transitions are taken into account. Since QWs are relatively shallow, simple selection rules based on the index of the subbands are not accurate, and overlap integrals between the calculated wavefunctions are used instead. The momentum matrix element is estimated based on the $\Gamma$-valley electron mass, the bandgap, and the spin-orbital splitting energy with a formula derived from $k \cdot p$ theory [66] and converted into the transition matrix elements for transverse electric (TE) and transverse magnetic (TM) fields [67] (Appendix B).

Lorentzian broadening of the gain spectrum is considered in the model to take into account interface roughness and alloy composition fluctuations [68] as well as carrier-carrier scattering [69,70]. Calculated gain spectra are convoluted with a Lorentzian function with unit area and an FWHM of $25 \mathrm{meV}$, consistent with the linewidth of low temperature, low excitation photoluminescence measurements taken on the MQW laser gain material [29] as well as typical values of 20 to $30 \mathrm{meV}$ reported and assumed for a range of material systems in the literature [69-71].

Calculated gain spectra are shifted to take bandgap narrowing (BGN) into account. A number of experimental studies have been made on the narrowing of the direct bandgap of n-type doped Ge [72,73]. However, since in Ge electrons are primarily in the L-valley, exchange and scattering interaction with $\Gamma$-valley electrons is minimal at low temperatures [73]. Thus, Ge is not representative here, and we evaluate BGN based on the exchange and correlation energy shifts of the Jain and Roulston model [74]. For $\Gamma$-electron concentrations of $\sim 2 \times 10^{17} \mathrm{~cm}^{-3}$, close to the threshold concentrations at $20 \mathrm{~K}$ and $50 \mathrm{~K}$, and corresponding hole concentrations of $\sim 4 \times 10^{17} \mathrm{~cm}^{-3}$ given the background doping, we estimate BGN at $24 \mathrm{meV}$. At increased temperatures, further BGN is expected as thresholds and carrier concentrations increase. At $100 \mathrm{~K}$ most of the additional electrons are in the L-valley, and BGN increases at a lower rate. Further details on the gain calculations can be found in Appendix B.

To convert material into modal gain, confinement factors are calculated for each of the QWs and for both the quasiTE and quasi-TM modes of the microdisk. Since both have TE and TM field components, separate confinement factors are calculated for the TE and TM material gain. The cylindrical geometry of the microdisk is taken into account by modifying the usual formula derived for rectilinear geometries [75] with conformal mapping [76], as also described in Appendix B. Higher-order modes are confined deeper inside the microdisk, further from its etched outer periphery, and have lower scattering losses as a consequence, so that they are also considered in the following.

Optical losses are modeled as the result of combined FCA and surface roughness induced scattering. FCA is modeled as a combination of intra-valley scattering as modeled with the Drude model [77] and IVBA [78] with transition matrix elements modeled with analytical expressions derived from sixband $k \cdot p$ theory as described in Appendix C. As for the gain calculations, FCA is calculated in all layers and weighted according to the optical mode overlaps.

The Drude model was evaluated separately for each carrier type with the mobilities already assumed in the transport modeling. Averaged in-plane and $z$-direction effective masses were assumed for TE and TM polarizations, respectively. The Drude model, relying on electrical mobilities rather than the corresponding quantities at optical transition energies, is an empirical description of FCA that requires corrections at optical frequencies [79]. Here, we apply a correction factor of $\sim 2$ to the electron FCA, as found in Ref. [80] for Ge, which might also be partially caused by intervalley transitions. For the valence band, data from Ge cannot be taken as a basis, as they are dominated by IVBA. For $\mathrm{Si}$, however, hole FCA follows the Drude model in the wavelength range of interest, also with a corrective coefficient of $\sim 2$, which we also take as a basis for our calculations.

IVBA is particularly strong in long-wavelength lasers, since the spin-orbit split-off (SO) band tends to have a larger energy separation $\Delta_{\mathrm{SO}}$ from the HH/LH bands in low bandgap semiconductors, with this energy separation approaching the bandgap energy at which the laser emits. In the gain materials utilized here, the SO-HH energy difference is in the 370 $400 \mathrm{meV}$ range, not far from the $494 \mathrm{meV}$ emission energy of the laser [29]. Since IVBA is also strongest close to $\Delta_{\text {SO }}$ [81], this leads to increased optical absorption.

Scattering losses [82] are modeled using 3D finite-difference time-domain (FDTD) simulations. A randomly generated surface roughness is implemented at the sidewalls of the underetched disk assuming the correlation length $L_{c}$ and the root mean square $\sigma$ of the roughness to be $500 \mathrm{~nm}$ and $55 \mathrm{~nm}$, respectively, as extracted from scanning electron micrographs of the microdisks. The quality $(Q)$ factor of the microdisk is extracted from the simulations and converted into linear propagation losses according to $\alpha=2 \pi n_{g} / Q \lambda$, with $n_{g}$ the group index and $\lambda$ the free-space wavelength. This results in $\alpha \simeq 52 \mathrm{~cm}^{-1}$ and $\alpha \simeq 44 \mathrm{~cm}^{-1}$ for TE and TM modes, respectively, with the higher TE-mode losses resulting from an increased surface overlap resulting from the continuity of the displacement (D-) field at the etched interface. While losses were calculated for TE and TM modes with radial mode numbers ranging from zero to five, each averaged over repeated simulations with different randomly generated roughness, the data were too scattered to identify significant trends over mode number and were thus averaged. The overall standard deviation of the simulated losses, as resulting from different roughness profiles, was $12 \mathrm{~cm}^{-1}$.

Parameters used for gain and loss calculations are further summarized in Table 2 .

\section{MODELING RESULTS}

The experimentally recorded pump intensity thresholds, after correction, are $16 \mathrm{~kW} / \mathrm{cm}^{2}, 20 \mathrm{~kW} / \mathrm{cm}^{2}$, and $146 \mathrm{~kW} / \mathrm{cm}^{2}$, respectively, at temperatures of $20 \mathrm{~K}, 50 \mathrm{~K}$, and $100 \mathrm{~K}$ at a $1064 \mathrm{~nm}$ excitation wavelength [29]. Threshold reduction relative to a DHS laser with thick $(380 \mathrm{~nm})$ gain material was attributed to a reduction in active material volume and in DOS inside the MQW. Moreover, dislocation networks were moved from the boundary of the active material in the DHS laser to below the buffer layer in the MQW laser, removing an important source of non-radiative recombination. 
The following modeling serves to better understand the remaining performance limitations of the MQW laser, in particular the very high temperature dependence of the lasing threshold past $50 \mathrm{~K}$. The impacts of factors such as dark recombination, finite directness, and low QW depths are analyzed in detail.

We first present the modeled modal net gain to verify and discuss the modeling assumptions and determine the lasing mode at threshold. A detailed discussion of modeling results in regard to carrier concentrations, optical gain, and optical loss is described thereafter and provides more detailed insight.

The net gain, which should be zero at threshold, is plotted as a function of the pump power density in Figs. 3(a) and 3(b) with different assumptions. This is shown for both the highest gain TE and TM modes. For TE polarization, this is the $\mathrm{TE}_{3}$ mode at both $50 \mathrm{~K}$ and $100 \mathrm{~K}$, whereas for TM polarization, this is $\mathrm{TM}_{3}$ at both $50 \mathrm{~K}$ and $100 \mathrm{~K}$ in Fig. 3(a), but $\mathrm{TM}_{3}$ and $\mathrm{TM}_{0}$ for $50 \mathrm{~K}$ and $100 \mathrm{~K}$ in Fig. 3(b). The subscript, starting from zero, denotes the radial mode number and corresponds to the number of nodes along the disk's radius. The switch between $\mathrm{TM}_{3}$ and $\mathrm{TM}_{0}$ for the highest gain $\mathrm{TM}$ mode, as the temperature is increased, results from their differing confinement factors. As shown in the following, at low temperatures, the balance between gain and losses favors TE polarization, as expected based on the small residual compressive strain in QWs. At $100 \mathrm{~K}$, however, TM modes undergo the highest net gain. While the quasi-TM $\mathrm{TM}_{0}$ mode has a much stronger TM confinement factor $\Gamma_{\mathrm{TM}}$ [see Eqs. (B8) and (B9) in Appendix B], the $\mathrm{TM}_{3}$ mode is mixed and actually has confinement factors that are predominantly $\mathrm{TE}$, with $\Gamma_{\mathrm{TE}}=0.257$ and $\Gamma_{\mathrm{TM}}=0.168$. This is a consequence of the quasi-TE and quasi-TM modes having strict TE/TM polarization only on the symmetry plane of the disk. The differences in net gain for the different radial mode numbers can however be very small, so that not too much importance should be given to the exact radial mode number. As we will see in the following, the data

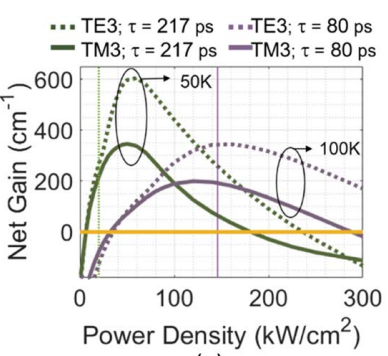

(a)

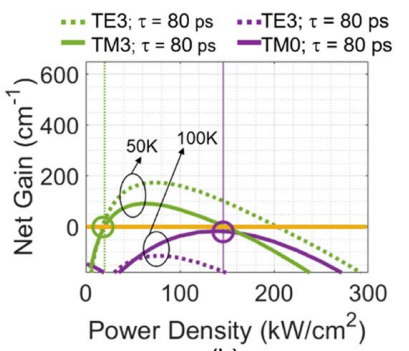

(b)
Fig. 3. Net gain as a function of pump power density. The experimentally determined thresholds are indicated by the vertical lines. (a) Lifetimes equal to $217 \mathrm{ps}$ and $80 \mathrm{ps}$ at $50 \mathrm{~K}$ and $100 \mathrm{~K}$, respectively. Positive net gains equal to $267 \mathrm{~cm}^{-1}$ and $342 \mathrm{~cm}^{-1}$ are observed at the experimental thresholds at $50 \mathrm{~K}$ and $100 \mathrm{~K}$, respectively. (b) Directness $\Delta E_{\mathrm{L}-\Gamma}$ reduced by $30 \mathrm{meV}$ and lifetimes uniformly set to $80 \mathrm{ps}$. The net gain at threshold is close to zero for both temperatures and rolls over at $100 \mathrm{~K}$. Pump power densities indicated on the $x$ axes can be converted into carrier concentrations averaged across the thin film stack by taking the assumed lifetimes and stack thickness $(690 \mathrm{~nm})$ into account. QW dependent carrier concentrations at threshold are reported in Fig. 6. is however conclusive in determining the polarization of the emission. The mode profiles and their polarization and QW-number dependent confinement factors are shown in Fig. 4. Tabulated optical simulation results can be found in the Supplementary Information in Ref. [65].

We assume initially a carrier lifetime of $217 \mathrm{ps}$, as measured by Julsgaard et al. [42] for a 12.5 at. \% GeSn alloy. Results do not appear to make physical sense, because at $50 \mathrm{~K}$, a large net gain of $267 \mathrm{~cm}^{-1}$ is calculated at the experimental threshold [Fig. 3(a)]. At $100 \mathrm{~K}$, we model a net gain curve that rolls over at about $50 \mathrm{~kW} / \mathrm{cm}^{2}$ (not shown), way before the experimental threshold of $146 \mathrm{~kW} / \mathrm{cm}^{2}$ that would be expected to be below the roll-over point. The maximum gain of $385 \mathrm{~cm}^{-1}$ is also very high at the temperature experimentally determined to be the maximum lasing temperature. All these indicate that the modeling parameters may be off their actual values.

Since $100 \mathrm{~K}$ is the maximum lasing temperature, we expect the net gain curve to reach a maximum close to zero and to roll over close to threshold, since any loss in gain due to increased temperature would then lead to lasing to cease. Reducing the assumed carrier lifetime to $80 \mathrm{ps}$ shifts the roll-over point of the $100 \mathrm{~K}$ gain curve to the experimental threshold, as shown by the $100 \mathrm{~K}$ net gain curve in Fig. 3(a); however, it does not substantially modify the maximum net gain. As already described above, lifetimes were measured in another experiment to be reduced from 435 ps to 170 ps as the Sn content is increased from 8.5 at. $\%$ to 12.5 at. \%. Assuming this to be driven primarily by bulk material quality and taking into account that concentrations above 14 at. \% are very difficult to grow with the growth process employed in Ref. [29], a reduction in carrier lifetime to 80 ps seems reasonable for the used 13.3 at. \% alloy.

A second discrepancy is seen in the high peak gain modeled at $100 \mathrm{~K}$. Modifying assumptions in regard to transition matrix elements for gain and IVBA calculations or in regard to FCA in a reasonable range did not resolve this discrepancy with experimental data. However, the maximum gain is also determined, among these other parameters, by the $\mathrm{L}-\Gamma$ energy difference $\Delta E_{\mathrm{L}-\Gamma}$. As the QWs fill, the electron quasi-Fermi level rises. Once it reaches the L-valley energy, the electron DOS suddenly increases, effectively clamping the electron Fermi level and limiting additional gain. Further optical pumping then contributes primarily to the L-valley and to electron mediated FCA. Since there remains some uncertainty on the bowing parameters determining $\Delta E_{\mathrm{L}-\Gamma}$, we also revisited the assumptions in this regard. Lowering $\Delta E_{\mathrm{L}-\Gamma}$ by $30 \mathrm{meV}$ and assuming the $80 \mathrm{ps}$ lifetime results in a net gain close to zero for both $50 \mathrm{~K}$ and $100 \mathrm{~K}$ temperatures [Fig. 3(b)]. Moreover, the net gain reaches a maximum at threshold at $100 \mathrm{~K}$ and rolls over thereafter, as expected.

The reduction in L-valley energy inside QWs is a tweaking parameter inside the model that could be associated to a number of physical effects. A reduction in the $\Gamma$-valley bowing parameter $b_{\Gamma}$ or an increase in the L-valley bowing parameter $b_{\mathrm{L}}$ by $0.26 \mathrm{eV}$ would account for a $30 \mathrm{meV}$ reduction in directness. While $b_{\Gamma}$ was obtained from experimental data [37] and is consistent with the values reported in other experimental studies [83], $b_{\mathrm{L}}$ is more difficult to directly experimentally measure and originated from a first-principle calculation [84]. 
(a)
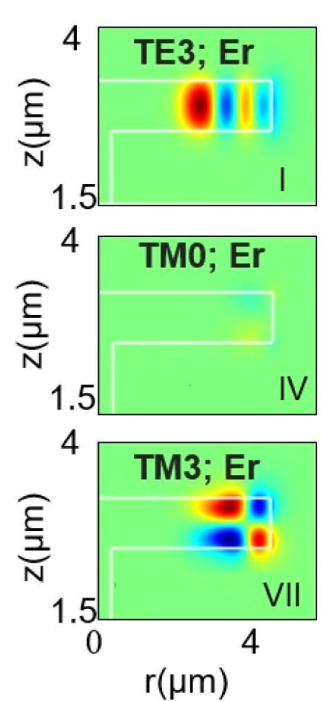
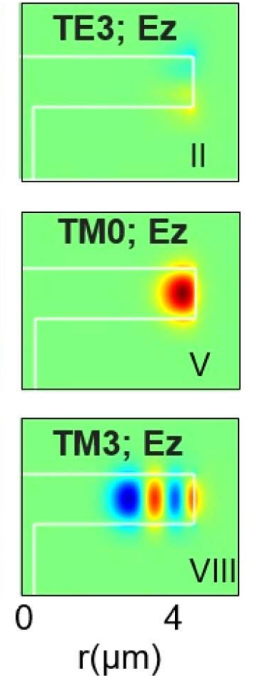

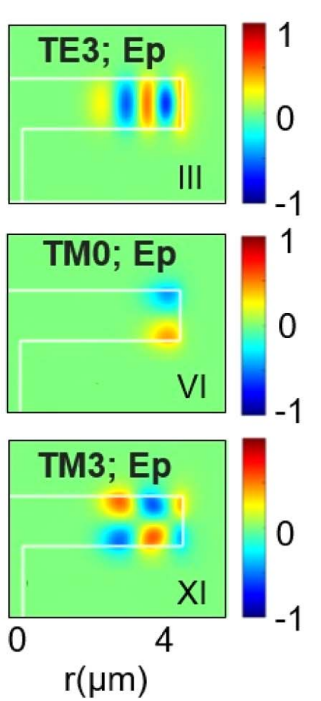

(b)

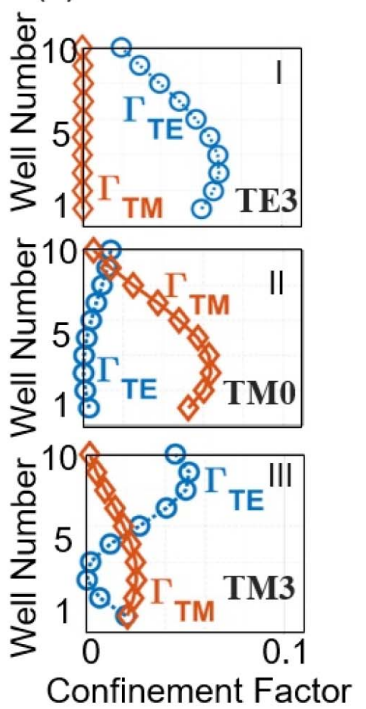

Fig. 4. (a) Field profiles for the $\mathrm{TE}_{3}, \mathrm{TM}_{0}$, and $\mathrm{TM}_{3}$ modes, with $E_{r}, E_{z}$, and $E_{p}$ the $E$-field components along the radial, $z$, and azimuthal directions. While $\mathrm{TM}_{3}$ is a quasi-TM mode, it also has strong in-plane field components $E_{r}$ and $E_{p}$ away from the disk's symmetry plane. This explains why it is the quasi-TM mode with the highest gain at low temperatures $(50 \mathrm{~K})$, at which the TE material net gain is the highest. At higher temperatures $(100 \mathrm{~K})$, the TM material net gain is highest, and $\mathrm{TM}_{0}$ becomes the dominant lasing mode. (b) TE and TM confinement factors $\Gamma_{\mathrm{TE}}$ and $\Gamma_{\mathrm{TM}}$ as a function of QW number for each of these three modes. The pronounced off-symmetry-plane $\Gamma_{\mathrm{TE}}$ for $\mathrm{TM}_{3}$ is readily apparent.

In addition, alloy composition fluctuations or collisional broadening might also contribute to effectively reducing the directness. Hot carrier effects, which may result from the high photon energy optical pump but are not modeled here, may also play a role in an increased L-valley population and carrier escape from QWs. An error in the $\Gamma$-valley electron effective mass towards higher values would lead to a slower filling of the QWs in simulations and the L-valley energies to be reached at a higher electron concentration. An offset in the assumed band alignments might lead to an increased leakage current from the QWs in experiments and to a higher electron population in the barrier layers. Finally, small errors in the assumed alloy composition or residual strain could also partially contribute to reduced directness. Consequently, this $30 \mathrm{meV}$ lowering of the L-valley energies should not be taken at face value, but rather as a strong indication that either directness or carrier confinement is overestimated in the uncorrected simulations.

While exact numbers for lifetime also cannot be extracted from these simulations, due to the large number of modeling assumptions, these results strongly indicate a lifetime in the 100 ps range as this was the only parameter that would extend the roll-over point of the net gain curve to threshold densities or above, as physically expected. Therefore, in the rest of this paper, we will assume the directness (reduced by $30 \mathrm{meV}$ ) and lifetime (80 ps) used to generate Fig. 3(b).

In our calculations, the peak net gain at $50 \mathrm{~K}$ is at a photon energy of $467 \mathrm{meV}, 27 \mathrm{meV}$ below what has been measured [29]. Besides a small error in the bowing parameters, this may also point to overestimation of $\mathrm{BGN}$, underestimation of the band filling (Moss-Burstein effect), or small errors in the assumed alloy composition or residual strain. Additionally, the approximation used in gain calculations, neglecting the wavevector dependent band mixing in transition matrix elements, also induces some discrepancy between calculated and measured gain peak positions.

At $100 \mathrm{~K}$, electron concentrations in the top QWs increase above $2 \times 10^{18} \mathrm{~cm}^{-3}$ as the L-valley gets populated. While L-valley electrons have a reduced effect on the narrowing of the optical bandgap as determined by $\Gamma$-valley energies, an additional $\mathrm{BGN}$ of $\sim 13 \mathrm{meV}$ is estimated for the high carrier concentrations seen at the $100 \mathrm{~K}$ threshold [73]. Taking this into account, we model the gain peak to be at a photon energy of $461 \mathrm{meV}$ at $100 \mathrm{~K}$, also $28 \mathrm{meV}$ below the experimentally recorded emission energy. In other words, the modeled relative changes in emission energy, as the laser temperature is changed, appear to be consistent with experiment.

At the lower temperature of $50 \mathrm{~K}$, the $\mathrm{TE}_{3}$ mode has a net gain only $22 \mathrm{~cm}^{-1}$ above that of the $\mathrm{TM}_{3}$ mode, making it difficult to pinpoint which one is the lasing mode given the remaining experimental and modeling uncertainties. On the other hand, at $100 \mathrm{~K}$, there is a clear distinction between the two polarizations' net gain. While the net gain for all TE modes stays negative and reaches a maximum of $-113 \mathrm{~cm}^{-1}$ for $\mathrm{TE}_{3}$, the $\mathrm{TM}_{0}$ mode reaches threshold at $140 \mathrm{~kW} / \mathrm{cm}^{2}$.

As seen in more detail in the following, lasing in TM modes can be explained by a number of factors. Even though $\mathrm{HHs}$ remain dominant, LH concentration is not much lower at the $100 \mathrm{~K}$ threshold, as a consequence of the high free carrier concentrations (that are themselves a consequence of L-valley filling) leading to valence band filling (due to charge neutrality) and to population of the LH band. Finally, the TE modes not only have more scattering losses as a consequence of their larger surface overlaps, but also suffer from increased IVBA as explained below.

Figure 5 shows modeling results assuming that the laser is pumped at threshold at $50 \mathrm{~K}$ and at $100 \mathrm{~K}$. Figure 5(a) shows 


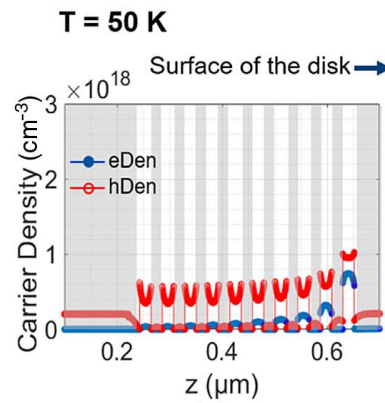

(a)

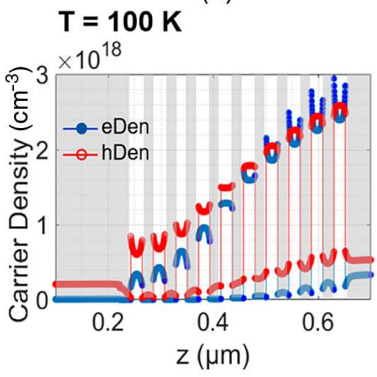

(d)

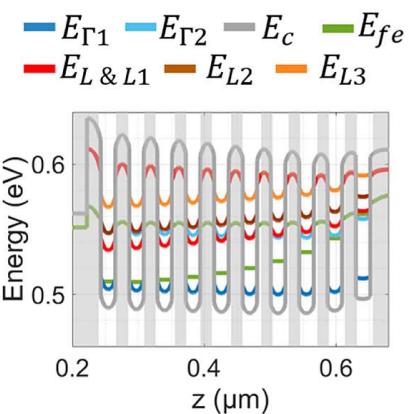

(b)

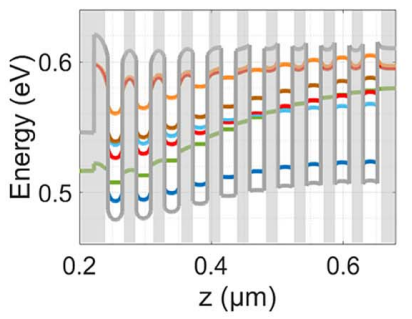

(e)
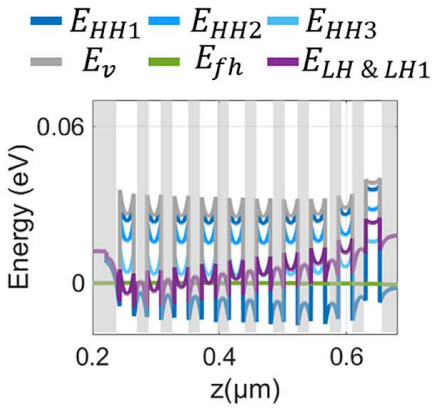

(c)

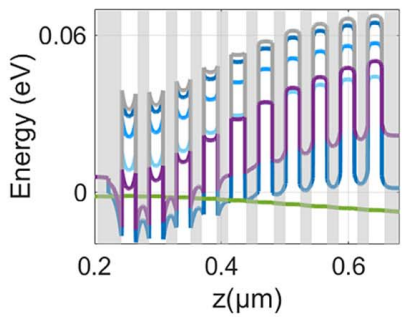

(f)

Fig. 5. Carrier concentrations versus vertical position for (a) $20 \mathrm{~kW} / \mathrm{cm}^{2}$ pump intensity at $50 \mathrm{~K}$ and (d) $150 \mathrm{~kW} / \mathrm{cm}^{2}$ pump intensity at $100 \mathrm{~K}$ along the axis indicated in Fig. 1. Barrier layers and buffer are shown as gray regions. Conduction and valence band energies at (b), (c) $50 \mathrm{~K}$ and (e), (f) $100 \mathrm{~K}$, with the same color coding as in Fig. 2. In addition, quasi-Fermi levels are shown in green. To reduce the number of curves, the red curves in (b) and (e) show the $E_{\mathrm{L}}$ band edge in the barriers, but the $E_{\mathrm{L} 1}$ subband edge in the wells. Similarly, the purple curves in (c) and (f) show $E_{\mathrm{LH}}$ in the barriers and $E_{\mathrm{LH} 1}$ in the wells.

electron and hole concentrations across the MQW stack at $50 \mathrm{~K}$. While electrons are well confined, it can be seen that holes already spill over as a consequence of their low confinement potential. Figures 5(b) and 5(c) show band diagrams, including the electron and hole quasi-Fermi levels $E_{f e}$ and $E_{f b}$, for the conduction and valence bands. It is apparent that in the topmost QW, the electron quasi-Fermi level is $50 \mathrm{meV}$ above the quantized edge of the first $\Gamma$-electron subband, so that close to maximum gain is obtained for this QW given the assumed $25 \mathrm{meV}$ gain broadening. Moreover, this gain cannot be increased much further as the Fermi level is right below the lowest L-valley energy and will thus be clamped as pump levels increase. The lower QWs, however, are only partially filled.

Figures 5(d)-5(f) show the electron/hole concentrations and corresponding band diagrams at the $100 \mathrm{~K}$ threshold. Carrier concentrations are significantly higher in the QWs, accounted for by L-valley population as shown in the following, and hole spillover being more pronounced. The electron quasiFermi level is clamped by the onset of the lowest L-valley subband for the six highest QWs, for which the hole quasi-Fermi level is also below the barrier energy. These QWs are thus at maximum capacity.

Further insight can be gained by classifying the carriers by type in each QW for temperatures $50 \mathrm{~K}$ and $100 \mathrm{~K}$. In Figs. 6(a) and 6(b), electron densities are reported in $\mathrm{cm}^{-2}$ for each of the QWs, resulting from the 2D DOS for bound states and from the integration across the QW thickness for unbound states. Carrier types are broken down into (total) $\Gamma$-valley $\left(n_{\Gamma}\right)$ and L-valley $\left(n_{\mathrm{L}}\right)$ electrons. Bound $\Gamma$-valley electrons with energies below the barrier energies are shown separately $\left(n_{\Gamma}^{\text {conf }}\right)$. Similarly, total hole densities are reported in Figs. 6(d) and 6(e) and classified as total $\mathrm{HHs}\left(p_{\mathrm{HH}}\right)$, total $\mathrm{LHs}\left(p_{\mathrm{LH}}\right)$, as well as a breakdown of confined $\mathrm{HHs}\left(p_{\mathrm{HH}}^{\text {conf }}\right)$ and $\mathrm{LHs}\left(p_{\mathrm{LH}}^{\text {conf }}\right)$.

At $50 \mathrm{~K}$, the carrier concentrations are relatively well behaved, in that electrons are predominantly confined $\Gamma$-valley electrons and holes are predominantly confined $\mathrm{HHs}$ [Figs. 6(a) and 6(d)]. Only the first QW, which receives the highest pump intensity, already features a large L-valley electron concentration resulting from the electron quasi-Fermi level reaching the L-valley energy [Fig. 5(b)]. Once this happens, the L-valley concentration rapidly grows due to its much higher DOS $(\sim 100 \times)$ resulting from both a higher DOS-mass $(\sim 8.4 \times)$ and its $4 \times$ degeneracy. Similarly, only the topmost QWs already feature a substantial amount of LHs as well as unconfined holes, resulting from the low barrier height for holes.

At $100 \mathrm{~K}$, the situation looks very different. Due to the L-valley pinning the Fermi levels, electrons remain confined in the QWs. However, due to increased carrier concentrations and thermalization, L-valley electrons, which contribute to FCA but not to gain, dominate. The lower QWs suffer not only from a reduced carrier concentration coming from lower pump levels, but also from earlier clamping of the $\Gamma$-electron concentration, resulting from the reduced directness due to the increased, residual compressive strain at these layers [Fig. 1(b)]. As a consequence, the top QWs are all filled to maximum capacity at the $100 \mathrm{~K}$ threshold, at which LHs reach similar levels as the HHs. Both types of holes are also largely unconfined, reducing the amount that interacts with the confined $\Gamma$-electrons (due to wavefunction overlap). 


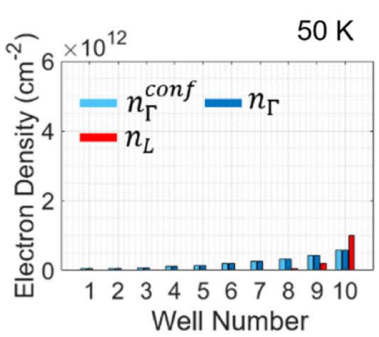

(a)

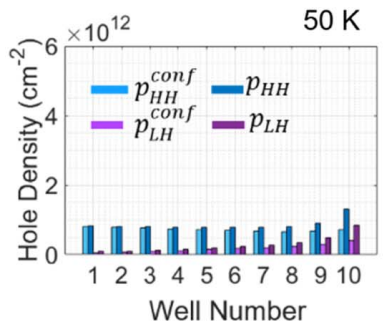

(d)

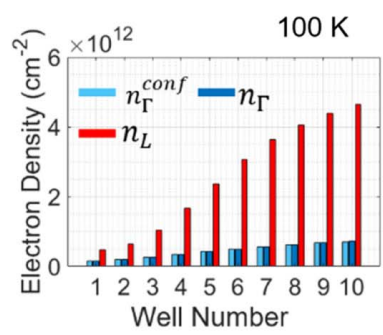

(b)

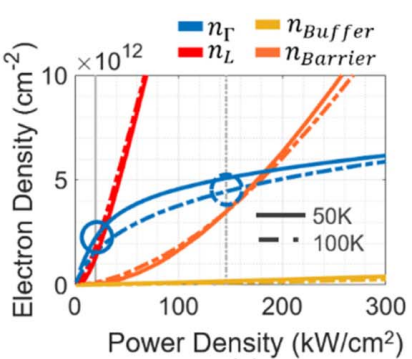

(c)

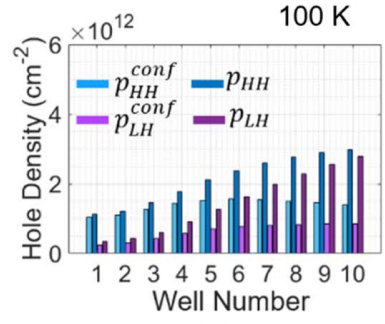

(e)

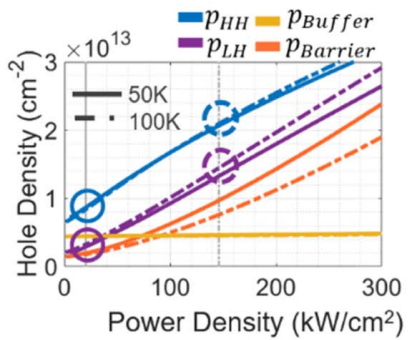

(f)

Fig. 6. (a), (b) Electron and (d), (e) hole concentrations for each of the wells at (a), (d) $50 \mathrm{~K}$ and at the $20 \mathrm{~kW} / \mathrm{cm}^{2}$ threshold pump intensity and (b), (e) $100 \mathrm{~K}$ and $150 \mathrm{~kW} / \mathrm{cm}^{2}$. Electrons are classified as (total) $\Gamma$ - and L-valley electrons, holes as (total) HHs and LHs. Confined $\Gamma$-valley electrons, HHs, and LHs are also separately shown. (c), (f) Carrier densities integrated over the entire stack and broken down in $\Gamma$ - and L-valley electrons inside the wells, total electron density in the barriers, and total electron density in the buffer. Similarly, holes are broken down in HHs and LHs inside the wells, total holes in the barriers, and total holes in the buffer.

The evolution of the carrier concentration with pump power intensities is summarized in Figs. 6(c) and 6(f). They show the concentrations of different carrier types integrated across the entire layer stack as a function of pump level for temperatures $50 \mathrm{~K}$ and $100 \mathrm{~K}$. Experimentally obtained lasing thresholds are marked by circles. Figure 6(c) distinguishes between the concentration of $\Gamma$-valley electrons in QWs $\left(n_{\Gamma}\right)$, L-valley electrons in QWs $\left(n_{\mathrm{L}}\right)$, electrons in the barriers $\left(n_{\text {Barrier }}\right)$, and electrons in the $\mathrm{Ge}_{0.90} \mathrm{Sn}_{0.10}$ buffer ( $\left.n_{\text {Buffer }}\right)$. Similarly, Fig. 6(f) distinguishes between the total concentration of $\mathrm{HHs}$ in QWs $\left(p_{\mathrm{HH}}\right)$, LHs in QWs $\left(p_{\mathrm{LH}}\right)$, holes in the barriers $\left(p_{\mathrm{Barrier}}\right)$, and holes in the buffer $\left(p_{\text {Buffer }}\right)$.

The clamping of the $\Gamma$-valley electrons and the continued growth of the L-valley concentration is clearly visible in Fig. 6(c). Moreover, the varying ratio between $\mathrm{HH}$ and $\mathrm{LH}$ concentrations can be seen in Fig. 6(f). The $n_{\mathrm{L}}$ curve is truncated by the limited range of the $y$ axis in Fig. 6(c), but reaches a value of $25 \times 10^{12} \mathrm{~cm}^{-2}$ at the $100 \mathrm{~K}$ threshold, $6 \times$ the $\Gamma$-valley electron density. To a lower extent $\left(0.8 \times n_{\Gamma}\right)$, population of the barriers also contributes to increasing the $100 \mathrm{~K}$ threshold. The buffer does not play a substantial role, with the free holes present there coming almost entirely from the intrinsic background doping.

From this analysis, the thermal sensitivity of the laser can be concluded to be primarily driven by electrons populating the L-valley. Once the Fermi level reaches the L-valley energy, the $\Gamma$-valley concentration can no longer be increased without a runoff of L-valley electrons and of the resulting FCA. This prevents further increase in gain and limits the ability of higher carrier concentrations to compensate for gain reduction caused by carrier thermalization as the laser temperature is further increased. As the L-valley electron concentration grows, hole concentrations also increase to maintain charge neutrality, resulting in a more isotropic gain (almost equal $\mathrm{HH}$ and $\mathrm{LH}$ concentrations) and a larger concentration of unconfined holes not substantially contributing to gain but increasing FCA. Notably, the QWs can be seen to fill up at the $100 \mathrm{~K}$ threshold, even though we assumed a quite low carrier lifetime, pointing to a lifetime in the 100 ps range not to be significantly underestimated.

While the low directness, combined with the reduced DOS and rapid filling of the QWs, appears to be the primary limitation here, our initial simulations with a higher directness (i.e., without the $30 \mathrm{meV}$ correction) were also limited by electron QW spill-over, as the L-valley energy was then slightly above the barrier energy for the topmost QWs. Thus, to reach efficient room temperature lasing with this structure, both directness and barrier height should be substantially increased. This poses the challenge of increasing the QW Sn content (higher directness) while simultaneously incorporating more silicon into the barriers [37].

Moreover, for both electrons and holes, the upper QWs have very different carrier concentrations than the bottom ones as a consequence of the decaying pump beam. In addition, $\Gamma$-valley concentrations in the lower barriers clamp earlier, due to reduced directness, caused by higher compressive strain, further limiting their capacity. Hence, this points to a reduction in the number of QWs to be beneficial for improving the pump thresholds and increasing the lasing temperature of this optically pumped structure, with performance increasing as carriers get more evenly distributed across the QWs [85].

Next, the modal gain per unit length is derived from the modeled carrier concentrations, as described in the previous section and in Appendix B. 


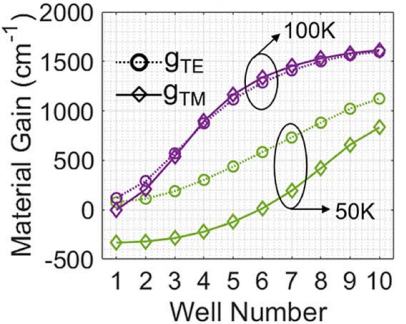

(a)

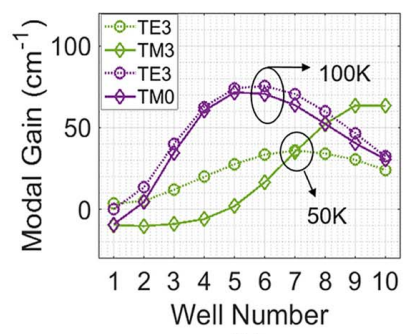

(b)

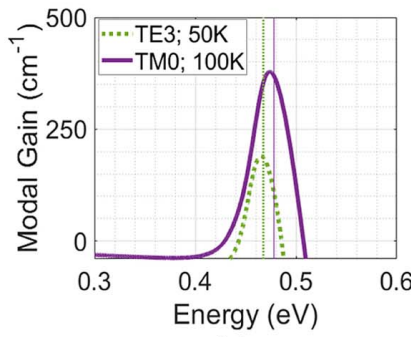

(c)

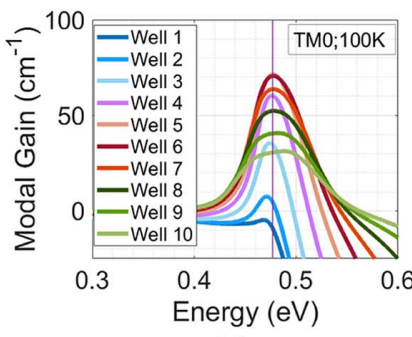

(d)

Fig. 7. Gain at $50 \mathrm{~K}$ and $100 \mathrm{~K}$, respectively, at $20 \mathrm{~kW} / \mathrm{cm}^{2}$ and $150 \mathrm{~kW} / \mathrm{cm}^{2}$ threshold pump intensities. (a) Material gain for each well, for both polarizations. (b) Modal gain for each well. The TE $3 / \mathrm{TM}_{3}$ modes have the highest gain at $50 \mathrm{~K}$, and the $\mathrm{TE}_{3} / \mathrm{TM}_{0} \mathrm{modes}$ at $100 \mathrm{~K}$. (c) Total modal gain of the $\mathrm{TE}_{3}$ and $\mathrm{TM}_{0}$ modes, respectively, at $50 \mathrm{~K}$ and $100 \mathrm{~K}$. (d) Breakdown of the modal gain for the $\mathrm{TM}_{0}$ mode at $100 \mathrm{~K}$ across quantum wells.

Figure 7 (a) shows the material gain at $50 \mathrm{~K}$ and $100 \mathrm{~K}$ for both polarizations and at each of the QWs. As expected, the top QW has the highest material gain for both polarizations, since it is exposed to the strongest pump beam intensity and has the highest directness. It is also apparent that while the TE material gain is higher at $50 \mathrm{~K}$, due to the dominant $\mathrm{HH}$ population, at $100 \mathrm{~K}, \mathrm{TE}$ and TM material gains are almost equal due to the redistribution between $\mathrm{HH}$ and $\mathrm{LH}$ populations.

The modal gains of the TE and TM modes with the highest net gain $\left(\mathrm{TE}_{3}\right.$ and $\mathrm{TM}_{3}$ at $50 \mathrm{~K}, \mathrm{TE}_{3}$ and $\mathrm{TM}_{0}$ at $\left.100 \mathrm{~K}\right)$ are shown as a function of the QW number in Fig. 7(b). It is derived from the material gain discussed above and the QW dependent modal confinement factors.

Due to the mixed polarization of the higher-order whispering gallery modes, some counterintuitive features can be seen in Fig. 7(b). For example, even though the TM material gain is lower than TE at $50 \mathrm{~K}$, the modal gain for the topmost QWs is higher for the $\mathrm{TM}_{3}$ mode. This results from $\mathrm{TM}_{3}$ having very strong TE-like field components away from the disk's centerplane (at QW No. 3 due to the underlying buffer), which are particularly strong in the topmost QWs as well as in the buffer (leading to excess absorption losses due to the low carrier population in the latter). In fact, the overall confinement factor of the $\mathrm{TM}_{3}$ mode is higher for TE than for TM, which explains why it features a comparatively high gain at $50 \mathrm{~K}$. On the other hand, the $\mathrm{TM}_{0}$ mode lasing at $100 \mathrm{~K}$ has clearly dominant $\mathrm{TM}$ confinement factors and is lasing due to high TM material gain [Fig. 7(a)] and reduced IVBA (see below).

Due to the presence of the $\mathrm{Ge}_{0.90} \mathrm{Sn}_{0.10}$ buffer at the bottom of the structure, the optical mode is pulled down relative to the MQW stack. As a consequence, the TE confinement factors of the $\mathrm{TE}_{3}$ mode are maintained between 0.049 and 0.069 for the seven bottommost QWs, but drop to 0.0039, 0.029, and 0.020 for the eighth, ninth, and tenth QWs, respectively. Similarly, the TM confinement factors of the $\mathrm{TM}_{0}$ mode are maintained between 0.037 and 0.064 for the seven bottommost QWs, but also drop to $0.025,0.014$, and 0.005 for the eighth, ninth, and tenth QWs. As a consequence, in most cases, the central QWs contribute most to the overall modal gain [with the exception of the $\mathrm{TM}_{3}$ gain at $50 \mathrm{~K}$, due to the strong role of the offcenter-plane TE confinement factors, Fig. 4(b)]. Here too, we see that the bottommost QWs could have been removed with very little loss in overall gain.
Figure 7(c) shows the total modal gain as a function of energy (not including the additional BGN occurring as the temperature is ramped up from $50 \mathrm{~K}$ to $100 \mathrm{~K}$ and the threshold increases accordingly). As the temperature is increased from $50 \mathrm{~K}$ to $100 \mathrm{~K}$, an increase in the peak modal gain from $189 \mathrm{~cm}^{-1}$ to $379 \mathrm{~cm}^{-1}$ can be observed. This increase is required to overcome additional FCA resulting from the population of the conduction $\mathrm{L}$-valleys and the resulting overall increase in carrier concentrations. As seen in Fig. 7(d), which shows the breakdown of the modal gain spectrum across QWs for $\mathrm{TM}_{0}$ at $100 \mathrm{~K}$, the QWs all have their peak gain close to the lasing energy. The overall spread in peak gain energies is relatively small and further reduced by the increased band filling of the top wells compensating for the bandgap reduction induced by the reduced compressive strain compared to the bottom wells.

Intra-valley FCA, as modeled with the Drude model including $2 \times$ empirical multiplicative correction factors, is first calculated for both polarizations at $50 \mathrm{~K}$ and $100 \mathrm{~K}$ and plotted in Fig. 8 (a). At $50 \mathrm{~K}$, it is evaluated as $55 \mathrm{~cm}^{-1}$ and $52 \mathrm{~cm}^{-1}$, respectively, for TE and TM modes, at the peak net gain photon energy. At $100 \mathrm{~K}$, this increases to $317 \mathrm{~cm}^{-1}$ and $261 \mathrm{~cm}^{-1}$, respectively.

The IVBA losses at $50 \mathrm{~K}$ are calculated as $55 \mathrm{~cm}^{-1}$ and $37 \mathrm{~cm}^{-1}$ [Fig. 8(b)] and increase to $226 \mathrm{~cm}^{-1}$ and $86 \mathrm{~cm}^{-1}$ at $100 \mathrm{~K}$, respectively. Noteworthily, IVBA losses are modeled as significantly larger for TE than for TM modes.

Based on the formulas derived in Appendix C, HHs contribute about $2 \times$ more to IVBA than LHs for TE polarization, while IVBA for TM polarization is almost fully dominated by LHs. This explains why IVBA is so much higher for TE polarization. Due to the large effective mass of $\mathrm{HHs}$ in the direction of quantization ( $z$ direction) and its large difference from the corresponding $\mathrm{SO}$ band effective mass, the three $\mathrm{HH}$ subbands span a large range of HH-SO transition energies. For each of the three corresponding values of $k_{z}$, the SO energy changes much more than the HH energy. On the other hand, as a consequence of the LH masses being much nearer to those of the SO band, the LH-SO transition energies are much more clustered around the transition energy at $k=0$, which is significantly below the emitted photon energy. As a consequence, the TE IVBA absorption distribution is broader in addition 


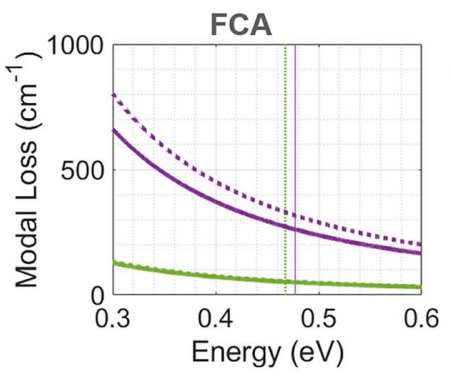

(a)

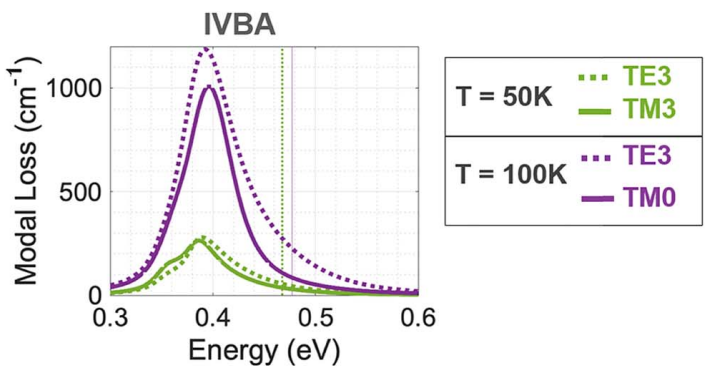

(b)

Fig. 8. Calculated absorption losses at $50 \mathrm{~K}$ and $100 \mathrm{~K}$ and at respective lasing thresholds $20 \mathrm{~kW} / \mathrm{cm}^{2}$ and $150 \mathrm{~kW} / \mathrm{cm}^{2}$. (a) FCA as predicted by the modified Drude model and (b) IVBA, calculated for both TE and TM polarizations.

to having a slightly larger peak value and maintains a larger value at the lasing energy.

Based on these numbers, FCA as predicted by the Drude model is a higher source of losses over IVBA. However, modeling indicates that IVBA plays a significant role in selecting TM modes at higher temperatures/higher threshold levels. While all three sources of losses are higher for TE, at $100 \mathrm{~K}$, the scattering losses, Drude model FCA, and IVBA are, respectively, $8 \mathrm{~cm}^{-1}, \sim 56 \mathrm{~cm}^{-1}$, and $\sim 140 \mathrm{~cm}^{-1}$ higher. Together, they significantly penalize the TE modes.

\section{DISCUSSION AND CONCLUSION}

The modeling performed in this paper indicates that the capacity of $\mathrm{SiGeSn} / \mathrm{GeSn} \mathrm{QW}$ s is limited primarily by the directness of the material clamping the Fermi level once it reaches the L-valley energy and, to a lesser extent by the shallowness of the QWs. Both are exacerbated by the low $\Gamma$-electron effective mass and reduced QW DOS, which lead to a rapid filling of the wells.

At $100 \mathrm{~K}$, the maximum achievable gain is reached at threshold and is limited by fully filled wells, as determined by Fermi-level clamping at the lowest L-valley subband energies. A high gain, on the order of $379 \mathrm{~cm}^{-1}$, appears to be required to overcome high FCA resulting from population of the conduction L-valleys and the accompanying increase in holes in addition to $\sim 44 \mathrm{~cm}^{-1}$ scattering losses. Lasing at higher temperatures is prevented by a runoff in FCA and the incapacity to compensate for it by further gain increase.

A larger L- to $\Gamma$-valley energy separation together with higher barriers would push out the FCA runoff to higher temperatures, by allowing increased $\Gamma$-electron concentrations to compensate for the gain reduction resulting from carrier thermalization, without incurring a resulting runoff in L-valley and barrier carrier concentrations. This requires increasing the $\mathrm{Sn}$ concentration inside the QWs, as well as the $\mathrm{Si}$ concentration inside the barriers, which has yet to be simultaneously achieved with high-quality growth. Recent results with $\mathrm{GeSn}$ lasers have confirmed that higher $S n$ content leads to higher lasing temperatures, but that lower Sn content alloys, grown at higher temperatures, are instrumental in reaching lower lasing thresholds, improved by lower defectivity and lower dark recombination. As an additional path towards jointly improving maximum lasing temperature and threshold, which are driven by the directness and the quality of the material, respectively, strain engineering in suspended microdisk lasers allows increasing the material directness at moderate Sn contents. This is why we are currently investigating concepts for electrically pumped GeSn microdisk lasers [40].

Incorporating $\mathrm{Si}$ into the barrier layers might also not be the only path forward towards QWs with both better confinement and better directness. $\mathrm{Ge}_{1-y} \mathrm{Sn}_{y} / \mathrm{Ge}_{1-x} \mathrm{Sn}_{x} / \mathrm{Ge}_{1-y} \mathrm{Sn}_{y} \mathrm{QWs}$ featuring type-I band alignment have been grown with $x=15$ at. \% and $y=9$ at. \% [86]. Growth of such structures might have some advantages, as the cracking of $\mathrm{Si}$ precursors is not required. The estimated reduction in bandgap between barrier and well, on the order of $100 \mathrm{meV}$ once the difference in strain of the pseudomorphically grown layers is taken into account, is, however, smaller than in the structure used here, which might be one of the reasons that this all-GeSn layer stack has not yet been shown to lase. A different band alignment and different partitioning of the confinement potentials between the valence and conduction bands, less favorable to the confinement of electrons, might also play a role in the other structure. Three-layer heterostructures with increasing $\mathrm{Sn}$ contents varying from 8 at. $\%$ in the bottommost layer to 17 at. $\%$ in the topmost layer have also been shown [87]. If sharp interfaces could be grown between a $\mathrm{Ge}_{0.83} \mathrm{Sn}_{0.17} \mathrm{QW}$ and $\mathrm{Ge}_{0.92} \mathrm{Sn}_{0.08}$ barriers on either side of the well, comparable confinement as seen here might be obtained with increased material directness.

The very low carrier lifetimes assumed here, on the order of 100 ps, appear consistent with the laser's properties. Moreover, modeling indicates that the directness of the material in the fabricated laser is lower than initially assumed, with a reduction in directness on the order of $30 \mathrm{meV}$ resulting in consistent modeling results (which corresponds to a valley separation between $\sim 40 \mathrm{meV}$ and $\sim 60 \mathrm{meV}$ across QWs). This corrective factor used in the model could, however, correspond to a number of physical causes and should be interpreted with caution.

Finally, due to the inhomogeneous QW filling observed here as a consequence of the decaying pump beam and the reducing well capacity as one moves deeper into the structure, it appears that reducing the number of QWs would be beneficial to improve the lasing threshold and maximum lasing temperature. In addition to providing insight into one specific device structure and $\mathrm{SiGeSn} \mathrm{MQW} \mathrm{lasers} \mathrm{in} \mathrm{general,} \mathrm{this} \mathrm{paper}$ describes a complete set of assumptions and a modeling flow for 
the predictive modeling of SiGeSn laser performance. A commercial semiconductor modeling tool has been adapted for solving transport equations and gain and loss calculations obtained by further post-processing of the data.

\section{APPENDIX A: TRANSPORT MODELING}

Modeling of valley-specific carrier concentrations is achieved by using the Multivalley model from sDevice in the bulk layers and the QWLocal quantization model in the wells. The Multivalley model allows individual specification of energy offsets from the band edge as well as DOS effective masses for each valley. Inside the QWs, the QWLocal model, along with considering several valence and conduction band valleys, also corrects the LDOS to take quantization effects into account. The LDOS is defined as the QWs' 2D DOS divided by the well width, whereafter the well is treated as if it were a $3 \mathrm{D}$ material. Corrections in the quantization models taking local fields in the wells into account [49] were turned off, as we found these to have very little effect on the high-level results, while this specific feature initially developed for the gallium nitride material system has not been validated for the application pursued here. Thus, quantization energies and quantized LDOS are identical to those of a square well with the same average barrier height in the absence of applied fields. This does not mean that fields inside the wells are fully ignored, as the drift-diffusion and Poisson equations are also solved inside the wells. In calculating the quantization energies, band intermixing effects are ignored, i.e., the quantization energies and LDOS are evaluated for each valley independently.

In the buffer and barrier layers, carrier concentrations are calculated according to 3D DOS using Fermi-Dirac statistics. Effective masses for the different valleys are defined with the Multivalley model. Since it is used only for evaluating the DOS, we directly plug in the DOS effective mass as obtained from a spherical average without loss of numerical accuracy.

QWs are modeled with the QWLocal model that uses the (e/h)Ladder $\left(m_{v, z}, m_{v, \|}, d_{v}, \Delta E_{v}\right)$ command in both well and barriers to define the energy and effective masses of the valley of index $v$, which stands for the $\Gamma$ - and $\mathrm{L}$-valleys for the conduction band and the $\mathrm{HH}$ and $\mathrm{LH}$ valleys for the valence band. $m_{v, z}$ is the effective mass along the direction of quantization, $m_{v, \|}$ the mass in the $x y$ plane, $d_{v}$ the band degeneracy, and $\Delta E_{v}$ the energy offset relative to the band edge. The ladder commands are required in both QW and barrier layers, as they are needed in the latter for correct calculation of quantization energies. Since we turn off the local field corrections by setting "MaxElecField" to zero, the quantization energies are calculated as those for a square well with the average barrier height defined by the (e/h)Ladder specifications of the two adjacent barrier materials. The electron density is then computed as

$$
n=\frac{k_{B} T}{L \hbar^{2} \pi} \sum_{v} d_{v} m_{v, \|} \sum_{j} F_{0}\left(\frac{E_{f e}-E_{C}-E_{v, j}}{k_{B} T}\right),
$$

where $L$ is the QW layer thickness, $\hbar$ the reduced Planck constant, $k_{B}$ the Boltzmann constant, $T$ the temperature, $E_{f e}$ the electron quasi-Fermi level, $E_{C}$ the local conduction band edge (as determined by the Poisson equation), and $E_{v, j}$ the quantized eigen-energy offset relative to the band edge for the $j$ th subband of a given valley of index $v$, which is independent of the position, as local field corrections have been turned off. $F_{0}$ is the complete Fermi-Dirac integral of index zero and corresponds to integrating the quantized DOS of the $j$ th subband times the occupation probability from the quantized eigenenergy to infinity. In other words, even for energies above the barrier energy, a finite number of quantized subbands continue to be assumed rather than reverting to the larger 3D DOS. Since only a finite number of actually quantized subbands, with eigenenergies below the barrier energies, are considered, this leads to an underestimate of the DOS. Most applications in other material systems have sufficiently deep QW for states above the barrier energy to have a low probability of occupation, so that this is usually a good approximation. Here, due to the relatively small confinement, it may lead to some modeling discrepancies at higher temperatures $(100 \mathrm{~K})$ at which the quasi-Fermi levels reach the tops of the wells for the valence band. Two subbands are considered for the $\Gamma$-valley electrons, two for L-valley electrons (three once the additional $30 \mathrm{meV}$ reduction in directness is considered), three for $\mathrm{HHs}$, and one for LHs. Since quasi-Fermi levels are exported from sDevice and carrier concentrations and energy distributions recalculated externally using MATLAB, it would have in principle been possible to recalculate them more rigorously including a 3D DOS inside the wells above the barrier energies. However, this would not necessarily increase the accuracy of the results, as it would result in carrier concentrations different from those entering the self-consistent solving of the drift-diffusion, thermionic current, and Poisson equations in sDevice. Thus, we have opted to evaluate the carrier concentrations with the same method as used in sDevice.

The thermionic emission model from sDevice is used to model the current at the heterojunction interfaces. Electron and hole current densities $J_{n}$ and $J_{p}$ are given by the following expressions, which can be reduced to the classic thermionic emission formula after some transformations:

$$
\begin{aligned}
& J_{n}=a_{n} q\left(v_{n, b} n_{b}-\frac{m_{n, b}}{m_{n, w}} v_{n, w} n_{w} e^{-\frac{\Delta E_{C}}{k_{B} T}}\right), \\
& J_{p}=-a_{p} q\left(v_{p, b} p_{b}-\frac{m_{p, b}}{m_{p, w}} v_{p, w} p_{w} e^{\frac{\Delta E_{V}}{k_{B} T}}\right),
\end{aligned}
$$

where $a_{n}, a_{p}$ are material dependent coefficients set in sDevice; $q$ is the elementary charge; $v_{n, b}, v_{n, w}, v_{p, b}, v_{p, w}$ are the electron and hole thermal velocities in the barrier and well; $n_{b}, n_{w}, p_{b}$, $p_{w}$ are the electron and hole concentrations in the barrier and well; $m_{n, b}, m_{n, w}, m_{p, b}, m_{p, w}$ are the electron and hole DOS effective masses in the barrier and well; and $\Delta E_{C}, \Delta E_{V}$ are the conduction and valence band discontinuities when transitioning from the well to the barrier material. The thermal velocities are given by

$$
v_{n / p, b / w}=\sqrt{\frac{k_{B} T}{2 \pi m_{n / p, b / w}}}
$$

and correspond to the mean thermal velocity projected onto the surface normal to the heterointerface, divided by two to take into account that only half the carriers propagate in the right direction to cross the interface. In the baseline sDevice models, 
when multivalley models are turned off and Boltzmann statistics are used, the carrier concentrations can be approximated as

$$
n / p_{b / w}=2\left(\frac{m_{n / p, b / w} \cdot k_{B} T}{2 \pi \hbar^{2}}\right)^{\frac{3}{2}} e^{\mp \frac{E_{C / V, b / w^{-}-E_{e} / h, b / w}}{k_{B} T}},
$$

so that the formulas for the thermionic currents reduce to

$$
\begin{aligned}
J_{n} & =a_{n} \frac{2 q m_{n, b} k_{B}^{2}}{(2 \pi)^{2} \hbar^{3}} T^{2}\left(e^{-\frac{E_{C, b^{-}} E_{f e, b}}{k_{B} T}}-e^{-\frac{E_{C, b}-E_{f e, w}}{k_{B} T}}\right) \\
& =a_{n} A_{n}^{*} T^{2}\left(e^{-\frac{E_{C, b}-E_{f e, b}}{k_{B} T}}-e^{-\frac{E_{C, b}-E_{f e, w}}{k_{B} T}}\right), \\
J_{p} & =-a_{p} \frac{2 q m_{p, b} k_{B}^{2}}{(2 \pi)^{2} \hbar^{3}} T^{2}\left(e^{+\frac{E_{V, b}-E_{f b, b}}{k_{B} T}}-e^{+\frac{E_{V, b}-E_{f b, w}}{k_{B} T}}\right) \\
& =-a_{p} A_{p}^{*} T^{2}\left(e^{+\frac{E_{V, b}-E_{f b, b}}{k_{B} T}}-e^{+\frac{E_{V, b^{-}-E_{f b, w}}}{k_{B} T}}\right),
\end{aligned}
$$

which are textbook equations for thermionic emission. $A_{n}^{*}$ and $A_{p}^{*}$ would be the Richardson constants if the barrier layer had isotropic effective masses $m_{n, b}$ and $m_{p, b}$. The parameters $a_{n}$ and $a_{p}$ serve to correct for the non-isotropy of the effective masses in sDevice [50].

Since we are using Fermi-Dirac statistics for evaluating the carrier concentrations and also activate them for the evaluation of the thermionic currents, the terms $\exp \left(\eta_{n / p, b / w}\right)$ are replaced by $\zeta\left(\eta_{n / p, b / w}\right)=\ln \left[1+\exp \left(-\eta_{n / p, b / w}\right)\right]+\eta_{n / p, b / w}$, with $\eta_{n, b / w}=-\left(E_{C, b}-E_{f e, b / w}\right) / k_{B} T$ and $\eta_{p, b / w}=\left(E_{V, b}-\right.$ $\left.E_{f h, b / w}\right) / k_{B} T$ as a correction to account for Fermi-Dirac statistics. The function $\zeta(\eta)$ has the property $\zeta(\eta) \simeq \exp (\eta)$ for large negative $\eta$, for which the Boltzmann statistics approximation is adequate, and $\zeta(\eta) \simeq \eta$ for large positive $\eta$, taking into account the Fermi blockade as bands fill.

In the case of anisotropic effective masses, the appropriate mass $m_{R}$ used for calculating the Richardson constant is the inplane mass multiplied by the degeneracy of the valley [50] and is given by

$$
\begin{gathered}
m_{R, \Gamma}=m_{\Gamma, \|, b}, \\
m_{R, \mathrm{~L}}=4 \cdot m_{\mathrm{L}, \|, b}=4 \cdot \sqrt{\frac{m_{\mathrm{L}, t, b}^{2}+2 m_{\mathrm{L}, l, b} m_{\mathrm{L}, t, b}}{3},} \\
m_{R, \mathrm{HH}}=m_{\mathrm{HH}, \|, b}, \\
m_{R, \mathrm{LH}}=m_{\mathrm{LH}, \|, b},
\end{gathered}
$$

where $m_{\mathrm{L}, l}$ and $m_{\mathrm{L}, t}$ are the longitudinal and transverse effective masses of the ellipsoidal L-valleys, respectively. Equation (A9) assumes the strain-induced splitting between the L-valleys to be small enough for all of them to contribute equally to the DOS, which is also the assumption made when defining the DOS with the multivalley sDevice model.

The DOS effective masses $m_{n}$ and $m_{p}$ used in Eqs. (A2)-(A7) are defined in sDevice with the commands (e/h)DOSMass and are used only for the modeling of thermionic currents, as the DOS itself is defined with the multivalley model (e/h)Valley. As explained in the following, their exact numerical value is irrelevant, as it is corrected by coefficients $a_{n}$ and $a_{p}$, which are calculated accordingly. Nonetheless, to use physically realistic numbers, we calculate them assuming simplified Boltzmann statistics to account for energy differences relative to the band edge as

$$
m_{n / p}=\left(\sum_{v} d_{v} m_{v}^{3 / 2} e^{\mp \frac{E_{v}-E_{C} / V}{k_{B} T}}\right)^{2 / 3}
$$

where the summation is taken over conduction or valence band valleys of index $v, d_{v}$ is the degeneracy of the valley of index $v$, $m_{v}$ is its effective DOS mass, and $E_{v}$ its energy. Coefficients $a_{n}$ and $a_{p}$ are then calculated as

$$
a_{n / p}=\frac{\sum_{v} m_{R, v} e^{\mp \frac{E_{v}-E_{C / V}}{k_{B} T}}}{m_{n / p}} .
$$

The exponential term in Eq. (A13) combines with the exponential terms in Eqs. (A6) and (A7) to shift the band edges $E_{C, b}$ and $E_{V, b}$ used in Eqs. (A6) and (A7) to the actual energy minimum of the valley. These coefficients are calculated in the simulation definition scripts and set with the "RegionInterface" commands. It should be noted that the methodology followed here corresponding to summing the Richardson constants for the different valleys assumes that carriers stay of the same type as they cross the barriers and ignores secondary effects associated with phonon or surface roughness scattering.

\section{APPENDIX B: GAIN CALCULATIONS}

The spatially varying quasi-Fermi levels are exported from sDevice and used to recover carrier concentrations and energy distributions for further use in gain and FCA calculations.

The material gain derived using Fermi's Golden Rule for each subband pair inside the QW can be written as [67]

$$
g_{21}^{\text {sub }}=\frac{\pi q^{2} \hbar}{n \varepsilon_{0} c m_{0}^{2} h \nu_{21}}\left|M_{T}\left(E_{21}\right)\right|^{2} \rho_{r}\left(E_{21}\right)\left(f_{2}-f_{1}\right) \text {, }
$$

where $q$ is the elementary charge, $\hbar$ the reduced Planck's constant, $n$ the refractive index, $\varepsilon_{0}$ the vacuum permittivity, $c$ the speed of light in vacuum, $m_{0}$ the free electron rest mass, $h$ Planck's constant, $\nu_{21}$ the photon frequency, and $\rho_{r}\left(E_{21}\right)$ the reduced DOS between two quantized subbands at the transition energy $E_{21}$. The Fermi factor $\left(f_{2}-f_{1}\right)$ accounts for the occupation probabilities, which are dependent on the optical pump intensities. Finally, $\left|M_{T}\right|^{2}$ refers to the transition matrix element that can be expressed in terms of the momentum matrix element $|M|^{2}$ according to $\left|M_{T}\right|^{2} /|M|^{2}=1 / 2(\mathrm{C}-\mathrm{HH})$ and $1 / 6(\mathrm{C}-\mathrm{LH})$ for TE and $0(\mathrm{C}-\mathrm{HH})$ and $2 / 3(\mathrm{C}-\mathrm{LH})$ for TM polarizations. $\mathrm{C}-\mathrm{HH}$ and $\mathrm{C}-\mathrm{LH}$, respectively, refer to conduction band to $\mathrm{HH}$ and conduction band to $\mathrm{LH}$ transitions.

Using $k \cdot p$ theory, $|M|^{2}$ can be theoretically related to the curvature of the conduction band and to the direct bandgap $E_{g}$ and spin-orbital splitting energy $\Delta_{\text {so }}$ and approximated as [66]

$$
|M|^{2}=\left(\frac{m_{0}}{m_{\Gamma}}-1\right) \frac{E_{g}+\Delta_{\text {so }}}{2\left(E_{g}+\frac{2}{3} \Delta_{\text {so }}\right)} m_{0} E_{g},
$$

with $m_{\Gamma}$ the conduction band effective mass at the $\Gamma$-valley. Note that Eq. (B2) underestimates $|M|^{2}$, e.g., in the case 
of GaAs, by about $26 \%$ [66]. The total gain is then found by summing over all possible valley and subband pairs:

$$
g_{21}\left(E_{21}\right)=\sum_{v_{c}, j_{c}} \sum_{v_{v}, j_{v}} g_{21}^{\mathrm{sub}}\left(v_{c}, j_{c}, v_{v}, j_{v}\right),
$$

where $v_{c}$ and $v_{v}$ index the conduction and valence band valleys, respectively, and $j_{c}$ and $j_{v}$ are the indices of their respective subbands.

In the following calculations, quantization energies for each subband are calculated under the assumption of no inter-band mixing taking place, i.e., states are assumed to remain pure $\mathrm{HH} / \mathrm{LH}$ band or conduction band $L / \Gamma$-valley states. However, finite barrier energies are taken into account in calculating the quantization energies. The reduced DOSs are evaluated with the textbook expression for $2 \mathrm{D}$ wells starting from the quantization energy up to infinity, with

$$
\rho_{r}\left(E_{21}\right)=\frac{m_{r, \|}}{\hbar^{2} \pi}
$$

and

$$
\frac{1}{m_{r, \|}}=\frac{1}{m_{v_{c, \|}}}+\frac{1}{m_{v_{v, \|}}},
$$

with $m_{r, \|}$ the reduced effective mass and $m_{v_{v, \|}}, m_{v_{c, \|}}$ the valence and conduction band effective masses, respectively, in the plane of the quantum well. This ignores the transition from a quantized 2D DOS to a 3D DOS as carrier energies reach the top of the wells. However, taking this into account proved to yield very small differences in the simulation results, so it was not further considered. This is also consistent with the way that carrier concentrations are evaluated from the quasi-Fermi levels as described in Appendix A.

The conversions between momentum and transition matrix elements described above also ignore inter-valley mixing inside the QWs. We do, however, take into account the overlap between the valence and conduction subbands, as given by the following corrective factor $\eta$ applied to the momentum matrix element:

$$
\eta=\frac{\left|\int_{z} \psi_{n_{c}} \psi_{n_{v}}^{*} \mathrm{~d} z\right|^{2}}{\int_{z} \psi_{n_{v}} \psi_{n_{\nu}}^{*} \mathrm{~d} z \int_{z} \psi_{n_{c}} \psi_{n_{c}}^{*} \mathrm{~d} z} .
$$

$\psi_{n_{v}}$ and $\psi_{n_{c}}$ are the slowly spatially varying envelopes of the wavefunctions obtained by imposing the boundary conditions corresponding to the continuity of $\psi$ (wavefunction continuity) and of $(\partial \psi / \partial z) \cdot 1 / m_{v, z}$ (particle flux conservation), with $m_{v, z}$ the effective mass of valley $v$ in the quantization direction $z$, at the QW boundary. This correction is particularly important for the laser modeled here, since strict selection rules based on the quantization index of the subbands would not be accurate due to the low barrier heights. For example, we estimate the $\Gamma_{1} \rightarrow \mathrm{HH}_{1}$ and $\Gamma_{2} \rightarrow \mathrm{HH}_{2}$ transitions to be, respectively, weakened to $96.7 \%$ and $89.2 \%$ of their uncorrected values (instead of being set to one), and the $\Gamma_{1} \rightarrow \mathrm{HH}_{3}$ transition to be corrected to $2.04 \%$ of its uncorrected value (instead of being set to zero).

To model linewidth broadening, $g_{21}$ is convoluted with a Lorentzian lineshape function $\mathcal{L}\left(h \nu_{0}-E_{21}\right)$ normalized to have a unit integral, with $h \nu_{0}$ the photon energy after convolution:

$$
g\left(h \nu_{0}\right)=\int g_{21}\left(E_{21}\right) \mathcal{L}\left(h \nu_{0}-E_{21}\right) \mathrm{d} E_{21} .
$$

The gain in the barrier layers and in the buffer is calculated following the same methodology. Since the barriers are not quantized, in principle the expression for the (3D) reduced DOS in a bulk material could have been used. However, to model the anisotropy of the effective masses as described here by the in-plane and $z$-direction effective masses $m_{v, \|}$ and $m_{v, z}$, the bulk materials were modeled as QWs with very large widths, for which the optical properties asymptotically tend to those of a bulk material as the width of the well is increased. Anisotropy of the effective masses for different directions inside the plane of the wells was, however, not taken into account.

The modal gain is calculated as $\Gamma_{\mathrm{TE}} g_{\mathrm{TE}}+\Gamma_{\mathrm{TM}} g_{\mathrm{TM}}$, with $\Gamma_{\mathrm{TE}}, \Gamma_{\mathrm{TM}}$ the confinement factors applicable to the material gains $g_{\mathrm{TE}}, g_{\mathrm{TM}}$ for pure TE and pure TM waves [75]. The confinement factor $\Gamma$ describes the overlap of the whispering gallery mode with the GeSn active material. We calculate it as [20]

$$
\begin{aligned}
\Gamma_{\mathrm{TE}} & =\frac{\iint_{\mathrm{GeSn}} n\left|E_{\|}\right|^{2} \frac{r}{R} \mathrm{~d} S}{Z_{0} \iint \vec{E} \times \vec{H} \cdot \vec{e}_{n} \cdot \mathrm{d} S}, \\
\Gamma_{\mathrm{TM}} & =\frac{\iint_{\mathrm{GeSn}} n\left|E_{z}\right|^{2} \frac{r}{R} \mathrm{~d} S}{Z_{0} \iint \vec{E} \times \vec{H} \cdot \vec{e}_{n} \cdot \mathrm{d} S},
\end{aligned}
$$

with $\vec{E}, \vec{H}$ the $E$ - and $H$-fields, $n$ the refractive index of GeSn, $E_{\|}, E_{z}$ the $E$-fields in the plane of the QW and in the quantization direction, $r$ the local radius in the cylindrical coordinate system, $R$ the radius of the disk, $Z_{0}$ the impedance of free space, $\vec{e}_{n}$ the unit vector normal to the disk's cross-section, and $\mathrm{d} S$ an infinitesimal surface element. The integral in the numerator is taken only over the GeSn QWs, whereas the one in the denominator is integrated over the transverse plane of the entire structure (relative to the direction of propagation of the mode). Light traveling closer to the periphery of the microdisk travels a longer distance in the active medium than light at a smaller local radius $r$; hence, it experiences a larger level of gain. This is captured by the term $r / R$, which is derived below from conformal mapping when transforming the cylindrical geometry into an equivalent rectilinear problem [76]. The transformation is indexed to the outer radius $R$ of the disk, as the round-trip gain is also calculated taking the circumference of the disk as a basis.

We start with the transformation $x+i y=R \cdot e^{\frac{u+i v}{R}}$, converting the spatial coordinates $x$ and $y$ into new coordinates $u$ and $v$, wherein $v / R$ is the angular coordinate in the cylindrical coordinate system. For a system with translation invariance in the $z$ direction, it can be shown that the wave equation in the original coordinate system given by

$$
\left(\Delta_{x y}+n^{2} k_{0}^{2}\right) E=0,
$$

with $\Delta_{x y}$ the Laplace operator in $x$ and $y, n$ the refractive index, and $k_{0}$ the magnitude of the wave-vector in free space, yields the same solutions as the transformed equation

$$
\left(\Delta_{u v}+n^{\prime 2} k_{0}^{2}\right) E=0,
$$

where the transformed refractive index $n^{\prime}$ is given by 


$$
n^{\prime}=e^{\frac{u}{R}} \cdot n=\left(\frac{x}{R} n\right)_{@ y=0} .
$$

Applying first-order perturbation theory to the wave equation in the transformed coordinate system, we obtain

$$
\operatorname{Im}\left(n_{\mathrm{eff}, u v}\right)=\frac{\iint_{\mathrm{GeSn}} \operatorname{Im}\left(e^{\frac{u}{R}} n\right) \operatorname{Re}\left(e^{\frac{u}{R}} n\right)|E|^{2} \mathrm{~d} u \mathrm{~d} z}{Z_{0} \iint \vec{E} \times \vec{H} \cdot \vec{e}_{n, u v} \cdot \mathrm{d} u \mathrm{~d} z},
$$

with $n_{\text {eff, } u v}$ the effective index and $\vec{e}_{n, u v}$ the normal vector of the equivalent rectilinear waveguide in the transformed coordinate system. Further using $\mathrm{d} u=e^{-\frac{u}{R}} \cdot \mathrm{d} x, \quad\left(\vec{e}_{n, u v}\right)_{@ y=0}=\vec{e}_{n, x y} \cdot \frac{x}{R}$, and $\left(e^{\frac{u}{R}}\right)_{@ y=0}=\frac{x}{R}$, we obtain

$$
\operatorname{Im}\left(n_{\mathrm{eff}}, u v\right)=\frac{\iint_{\mathrm{GeSn}} \operatorname{Im}(n) \operatorname{Re}(n)|E|^{2} \frac{x}{R} \mathrm{~d} x \mathrm{~d} z}{Z_{0} \iint \vec{E} \times \vec{H} \cdot \vec{e}_{n, x y} \cdot \mathrm{d} x \mathrm{~d} z} .
$$

By definition, $4 \pi \operatorname{Im}\left(n_{\mathrm{eff}}, u v\right) / \lambda_{0}$ describes the gain of the disk as referenced relative to the travel path along its circumference, at radius $R$, so that we recover Eqs. (B8) and (B9). Since the disk is not translation invariant in the $z$ direction, applying this simple form of conformal mapping is approximate. It constitutes, however, a good first-order correction to the usual formula of the confinement factor.

\section{APPENDIX C: MODELING OF INTERVALENCE BAND ABSORPTION}

IVBA is modeled following a similar methodology as for the gain. Equation (B1) is again applied, with a few refinements. For one, the transition matrix element for IVBA is $k$-vector and thus photon energy dependent [78]. This stems from the fact that it results from the s-orbital-like components of the SO band states, which increase as one moves away from the $\Gamma$-valley (and are zero at the $\Gamma$-valley due to symmetry), resulting in a transition matrix element $\left|M_{T}\left(E_{21}\right)\right|^{2}$ scaling as $k^{2}$ around $k=0$. A second aspect results from the fact that the in-plane masses for the $\mathrm{HH}$ are lower than for the SO band, so that resulting absorption peaks for $\mathrm{HH}$ start from the $\mathrm{HH}-\mathrm{SO}$ energy difference at $k=0$ and move further to lower, rather than to higher, absorption energies as the magnitude of the $k$-vector is increased.

Finally, inside the QWs, we had to take into account that the $\mathrm{HH}$ and $\mathrm{LH}$ are confined, while the SO holes have energies below the valence band barrier energies and are thus unconfined. To model this, we first take a Fourier transform of the $\mathrm{HH}$ and $\mathrm{LH}$ wavefunction envelopes, as previously described, to determine a weighted range of SO band states with which they interact. This effectively leads to an additional spectral broadening as compared to the absorption spectrum as calculated assuming confined SO band holes. As for the gain, IVBA of the barrier and buffer layers is calculated by modeling the material as a very wide QW, in which case strict selection rules could be assumed without loss of numerical accuracy.

For the gain calculations, evaluation of Eq. (B1) was followed by a convolution with the Lorentzian lineshape function accounting for carrier-carrier scattering, composition fluctuations, and surface roughness.

Analytical expressions for the transition matrix elements for IVBA are derived following the formalism of six-band $k \cdot p$ theory. Six-band $k \cdot p$ theory is chosen here rather than eight-band $k \cdot p$ theory, as its Hamiltonian treats only the six valence bands explicitly, while mixing of valence band states with s-like conduction band states - which is of paramount importance for the evaluation of IVBA - is implicitly accounted for by the wavefunction basis. This will be used in the following.

The valence band wavefunctions can be decomposed in a basis expressed in terms of their $\Gamma$-valley counterparts, to the first order in terms of the $k \cdot p$ perturbation, as [88]

$$
|s, \nu, \boldsymbol{k}\rangle=e^{i \boldsymbol{k} \cdot \boldsymbol{r}}\left(|s, \nu\rangle+\frac{\hbar}{m_{0}} \sum_{l} \frac{\left\langle u_{l}|\boldsymbol{k} \cdot \boldsymbol{p}| s, \nu\right\rangle}{E_{0}-E_{l}}\left|u_{l}\right\rangle\right),
$$

where $\boldsymbol{k}$ is the $k$-vector and $|s, \nu\rangle=|s, \nu, \mathbf{0}\rangle$ the valence band wavefunction at $\boldsymbol{k}=\mathbf{0}$, with total angular momentum $s$ taking the value $\nu$ after projection on the $z$ axis. $E_{0}$ is the energy of the valence band minimum. The summation over states $\left|u_{l}\right\rangle$ with energy $E_{l}$ accounts for mixing with all other states other than with the six valence bands, which are excluded from the summation, and also includes the conduction bands with their s-orbital-like states. $\boldsymbol{p}$ is the momentum operator. At $\boldsymbol{k}=\mathbf{0}$, $s=3 / 2, \quad v= \pm 3 / 2$ correspond to HHs, $s=3 / 2$, $v= \pm 1 / 2$ to LHs and $s=1 / 2, v= \pm 1 / 2$ to the SO bands. In this basis, the six-band Hamiltonian takes the form [89]

$$
\begin{aligned}
H_{i j}= & {\left[E_{j}(0)+\frac{\hbar^{2} k^{2}}{2 m_{0}}\right] \delta_{i j} } \\
& +\frac{\hbar^{2}}{m_{0}^{2}} \sum_{\alpha, \beta} k_{\alpha} k_{\beta} \sum_{l} \frac{\left\langle i, \mathbf{0}\left|p_{\alpha}\right| u_{l}\right\rangle\left\langle u_{l}\left|p_{\beta}\right| j, \mathbf{0}\right\rangle}{E_{0}-E_{l}},
\end{aligned}
$$

with $i, j$ indexing one of the six valence basis states with energy $E_{j}(0)$ at $\boldsymbol{k}=\mathbf{0}$ and $\alpha, \beta$ indexing the three spatial coordinates. It can be written in matrix form in terms of the (unmodified) Luttinger parameters used in six-band $k \cdot p$ theory [89] as

$$
H=-\left[\begin{array}{cccccc}
P+Q & -S & R & 0 & -S / \sqrt{2} & \sqrt{2} R \\
-S^{+} & P-Q & 0 & R & -\sqrt{2} Q & \sqrt{3 / 2} S \\
R^{+} & 0 & P-Q & S & \sqrt{3 / 2} S^{+} & \sqrt{2} Q \\
0 & R^{+} & S^{+} & P+Q & -\sqrt{2} R^{+} & -S^{+} / \sqrt{2} \\
-S^{+} / \sqrt{2} & -\sqrt{2} Q^{+} & \sqrt{3 / 2} S & -\sqrt{2} R & P+\Delta_{\text {so }} & 0 \\
\sqrt{2} R^{+} & \sqrt{3 / 2} S^{+} & \sqrt{2} Q^{+} & -S / \sqrt{2} & 0 & P+\Delta_{\text {so }}
\end{array}\right],
$$


with $\Delta_{\text {so }}$ the spin-orbit splitting energy, and

$$
\begin{aligned}
& P=\frac{\hbar^{2}}{2 m_{0}} \gamma_{1}\left(k_{x}^{2}+k_{y}^{2}+k_{z}^{2}\right), \\
& Q=\frac{\hbar^{2}}{2 m_{0}} \gamma_{2}\left(k_{x}^{2}+k_{y}^{2}-2 k_{z}^{2}\right), \\
& R=\frac{\hbar^{2}}{2 m_{0}}\left[-\sqrt{3} \gamma_{2}\left(k_{x}^{2}-k_{y}^{2}\right)+i 2 \sqrt{3} \gamma_{3} k_{x} k_{y}\right], \\
& S=\frac{\hbar^{2}}{2 m_{0}} 2 \sqrt{3} \gamma_{3}\left(k_{x}-i k_{y}\right) k_{z},
\end{aligned}
$$

in which the basis has been ordered as $|3 / 2,-3 / 2, \boldsymbol{k}\rangle$, $|3 / 2,-1 / 2, \boldsymbol{k}\rangle,|3 / 2,1 / 2, \boldsymbol{k}\rangle,|3 / 2,3 / 2, \boldsymbol{k}\rangle,|1 / 2,-1 / 2, \boldsymbol{k}\rangle$, $|1 / 2,1 / 2, \boldsymbol{k}\rangle$.

In the following, we will equate the wavefunctions of the $\mathrm{HH}, \mathrm{LH}$, and $\mathrm{SO}$ bands to $|3 / 2, \pm 3 / 2, \boldsymbol{k}\rangle,|3 / 2, \pm 1 / 2, \boldsymbol{k}\rangle$, and $|1 / 2, \pm 1 / 2, \boldsymbol{k}\rangle$, respectively, ignoring further mixing among these basis states at non-zero $\boldsymbol{k}$ as resulting from the Hamiltonian Eq. (C3). While this would be a rather coarse approximation in an unstrained bulk material, for the gain materials investigated in this paper, energy splitting resulting from both strain and quantization removes the degeneracy and suppresses this mixing, so we expect this to be an adequate approximation here. In eight-band $k \cdot p$ theory, in which the conduction band is also explicitly treated by the Hamiltonian, such an approximation would be entirely inadequate as a consequence of mixing of the valence bands with s-like orbital states being ignored. Here, however, mixing of the basis states with the s-orbital-like conduction bands is implicitly taken into account due to the conduction bands being part of the summation over $l$ in Eq. (C1).

From the basis states (now wavefunctions) described by Eq. (C1), the intervalence band transition matrix elements are derived as

$$
\begin{aligned}
M_{T} & =\langle i, \boldsymbol{k}|\boldsymbol{e} \cdot \boldsymbol{p}| j, \boldsymbol{k}\rangle \\
& =\sum_{\alpha, \beta} e_{\alpha} \hbar k_{\beta}\left(\delta_{i j} \delta_{\alpha, \beta}+\frac{2}{m_{0}} \sum_{l} \frac{\left\langle i, \mathbf{0}\left|p_{\alpha}\right| u_{l}\right\rangle\left\langle u_{l}\left|p_{\beta}\right| j, \mathbf{0}\right\rangle}{E_{0}-E_{l}}\right),
\end{aligned}
$$

where $\boldsymbol{e}$ is a unit vector indicating the polarization of light. This expression can be evaluated in terms of the Hamiltonian matrix elements by evaluating the terms $\sum_{l}\left\langle i, \mathbf{0}\left|p_{\alpha}\right| u_{l}\right\rangle\left\langle u_{l}\left|p_{\beta}\right| j, \mathbf{0}\right\rangle /$ $\left(E_{0}-E_{l}\right)$ with Eq. (C2) and equating Eqs. (C2) and (C3). In particular, we obtain

$$
\begin{aligned}
& \sum_{l} \frac{\left\langle 1\left|p_{\alpha}\right| u_{l}\right\rangle\left\langle u_{l}\left|p_{\beta}\right| 6\right\rangle}{E_{0}-E_{l}} \\
& =\frac{m_{0}}{\sqrt{2}}\left[\sqrt{3} \gamma_{2}\left(\delta_{\alpha x} \delta_{\beta x}-\delta_{\alpha y} \delta_{\beta y}\right)-i \sqrt{3} \gamma_{3}\left(\delta_{\alpha x} \delta_{\beta y}+\delta_{\alpha y} \delta_{\beta x}\right)\right],
\end{aligned}
$$

$$
\begin{aligned}
\sum_{l} \frac{\left\langle 2\left|p_{\alpha}\right| u_{l}\right\rangle\left\langle u_{l}\left|p_{\beta}\right| 6\right\rangle}{E_{0}-E_{l}}= & -\frac{m_{0}}{2 \sqrt{2}} 3 \gamma_{3}\left(\delta_{\alpha x} \delta_{\beta z}+\delta_{\alpha z} \delta_{\beta x}\right. \\
& \left.-i \delta_{\alpha y} \delta_{\beta z}-i \delta_{\alpha z} \delta_{\beta y}\right),
\end{aligned}
$$

$$
\begin{gathered}
\sum_{l} \frac{\left\langle 3\left|p_{\alpha}\right| u_{l}\right\rangle\left\langle u_{l}\left|p_{\beta}\right| 6\right\rangle}{E_{0}-E_{l}} \\
=-\frac{m_{0}}{\sqrt{2}} \gamma_{2}\left(\delta_{\alpha x} \delta_{\beta x}+\delta_{\alpha y} \delta_{\beta y}-2 \delta_{\alpha z} \delta_{\beta z}\right), \\
\sum_{l} \frac{\left\langle 4\left|p_{\alpha}\right| u_{l}\right\rangle\left\langle u_{l}\left|p_{\beta}\right| 6\right\rangle}{E_{0}-E_{l}} \\
=\frac{m_{0}}{2 \sqrt{2}}\left[\sqrt{3} \gamma_{3}\left(\delta_{\alpha x} \delta_{\beta z}+\delta_{\alpha z} \delta_{\beta x}+i \delta_{\alpha y} \delta_{\beta z}+i \delta_{\alpha z} \delta_{\beta y}\right)\right] .
\end{gathered}
$$

The transition matrix element for the HH to SO band transitions, averaged over all polarizations, can be evaluated by squaring Eqs. (C5) and Eq. (C8), taking the expectation values of $e_{\alpha}^{2}$ to be $1 / 3$ for all three spatial directions, and summing them to take both transitions given the SO band degeneracy into account. The same procedure is followed for $\mathrm{LH}$ to SO transitions by using Eqs. (C6) and (C7). This then results in

$$
\begin{aligned}
& \sum_{i=\{1\}, j=\{5,6\}}|\langle i|\boldsymbol{e} \cdot \boldsymbol{p}| j\rangle|_{\mathrm{HH} \rightarrow \mathrm{SO}}^{2} \\
& =2 \hbar^{2}\left(\gamma_{2}^{2}+\gamma_{3}^{2}\right)\left(k_{x}^{2}+k_{y}^{2}\right)+\frac{\hbar^{2}}{2} \gamma_{3}^{2}\left(k_{z}^{2}+k^{2}\right), \\
& \left.\sum_{i=\{2\}, j=\{5,6\}}\langle i|\boldsymbol{e} \cdot \boldsymbol{p}| j\rangle\right|_{\mathrm{LH} \rightarrow \mathrm{SO}} ^{2} \\
& =\frac{2}{3} \hbar^{2} \gamma_{2}^{2}\left(3 k_{z}^{2}+k^{2}\right)+\frac{3 \hbar^{2}}{2} \gamma_{3}^{2}\left(k_{z}^{2}+k^{2}\right) .
\end{aligned}
$$

Instead of evaluating the transitions for an average polarization, an averaged in-plane TE polarization or TM polarization can also be considered, corresponding, respectively, to taking the expectation values $e_{1}^{2}=e_{2}^{2}=1 / 2$ and $e_{3}^{2}=0$ for TE and $e_{1}^{2}=e_{2}^{2}=0$ and $e_{3}^{2}=1$ for TM. These then result in

$$
\begin{gathered}
\left.\sum_{i=\{1\}, j=\{5,6\}}\langle i|\boldsymbol{e} \cdot \boldsymbol{p}| j\rangle\right|_{\mathrm{HH} \rightarrow \mathrm{SO}, \mathrm{TE}} ^{2} \\
=3 \hbar^{2}\left(\gamma_{2}^{2}+\gamma_{3}^{2}\right)\left(k_{x}^{2}+k_{y}^{2}\right)+\frac{3}{2} \hbar^{2} \gamma_{3}^{2}\left(k_{z}^{2}\right), \\
\left.\sum_{i=\{2\}, j=\{5,6\}}\langle i|\boldsymbol{e} \cdot \boldsymbol{p}| j\rangle\right|_{\mathrm{LH} \rightarrow \mathrm{SO}, \mathrm{TE}} ^{2} \\
=\hbar^{2} \gamma_{2}^{2}\left(k_{x}^{2}+k_{y}^{2}\right)+\frac{9}{2} \hbar^{2} \gamma_{3}^{2}\left(k_{z}^{2}\right), \\
\left.\sum_{i=\{1\}, j=\{5,6\}}\langle i|\boldsymbol{e} \cdot \boldsymbol{p}| j\rangle\right|_{\mathrm{HH} \rightarrow \mathrm{SO}, \mathrm{TM}} ^{2}=\frac{3 \hbar^{2}}{2} \gamma_{3}^{2}\left(k_{x}^{2}+k_{y}^{2}\right), \\
\left.\sum_{i=\{2\}, j=\{5,6\}}\langle i|\boldsymbol{e} \cdot \boldsymbol{p}| j\rangle\right|_{\mathrm{LH} \rightarrow \mathrm{SO}, \mathrm{TM}} ^{2} \\
=8 \hbar^{2} \gamma_{2}^{2}\left(k_{z}^{2}\right)+\frac{9 \hbar^{2}}{2} \gamma_{3}^{2}\left(k_{x}^{2}+k_{y}^{2}\right) .
\end{gathered}
$$

Equations (C11)-(C14) are used to evaluate IVBA in this work. For any of the isotropic, TE or TM polarization cases, we can average $\left|M_{T}\right|^{2}$ for HHs and LHs and over all possible $k$-vector directions. We then obtain in all cases 


$$
\left|M_{T}\right|_{\text {av. }}^{2}=2 \hbar^{2} \gamma_{3}^{2} k^{2}+\frac{4}{3} \hbar^{2} \gamma_{2}^{2} k^{2}
$$

Transition matrix elements averaged over $k$-vector directions for the $\mathrm{HH} \rightarrow \mathrm{SO}$ and $L H \rightarrow \mathrm{SO}$ transitions, for TE and TM polarizations, are given by

$$
\begin{gathered}
\left|M_{T}\right|_{\mathrm{av}, \mathrm{HH} \rightarrow \mathrm{SO}, \mathrm{TE}}^{2}=2 \hbar^{2}\left(\gamma_{2}^{2}+\gamma_{3}^{2}\right) k^{2}+\frac{1}{2} \hbar^{2} \gamma_{3}^{2} k^{2}, \\
\left|M_{T}\right|_{\mathrm{av}, \mathrm{LH} \rightarrow \mathrm{SO}, \mathrm{TE}}^{2}=\frac{2}{3} \hbar^{2} \gamma_{2}^{2} k^{2}+\frac{3}{2} \hbar^{2} \gamma_{3}^{2} k^{2}, \\
\left|M_{T}\right|_{\mathrm{av}, \mathrm{HH} \rightarrow \mathrm{SO}, \mathrm{TM}}^{2}=\hbar^{2} \gamma_{3}^{2} k^{2}, \\
\left|M_{T}\right|_{\mathrm{av}, \mathrm{LH} \rightarrow \mathrm{SO}, \mathrm{TM}}^{2}=\frac{8}{3} \hbar^{2} \gamma_{2}^{2} k^{2}+3 \hbar^{2} \gamma_{3}^{2} k^{2} .
\end{gathered}
$$

Given the Luttinger parameters assumed for the gain medium $\left(\gamma_{2}=7.36, \quad \gamma_{3}=8.80\right)$, we find that $\left|M_{T}\right|_{\mathrm{av}, \mathrm{HH} \rightarrow \mathrm{SO}, \mathrm{TE}}^{2} \simeq 2 \cdot\left|M_{T}\right|_{\mathrm{av}, \mathrm{LH} \rightarrow \mathrm{SO}, \mathrm{TE}}^{2},\left|M_{T}\right|_{\mathrm{av}, \mathrm{LH} \rightarrow \mathrm{SO}, \mathrm{TM}}^{2} \simeq$ 4.9 $\left|M_{T}\right|_{\text {av. }, \mathrm{HH} \rightarrow \mathrm{SO}, \mathrm{TM}}^{2}$. It can thus be seen that HHs contribute $\sim 2 \times$ more than LHs to IVBA for TE polarization, while IVBA for TM polarization is very strongly dominated by LHs. Expressions consistent with Eqs. (C11)-(C14) were found to be derived in Ref. [90] in a different context.

The (unmodified) Luttinger parameters $\gamma_{1}, \gamma_{2}, \gamma_{3}$ are extracted from data reported in Ref. [47] and used directly to evaluate the expressions of the transition matrix elements derived here from six-band $k \cdot p$ theory. Other calculations made in this paper with eight-band $k \cdot p$ theory are based on the corresponding modified Luttinger parameters $\bar{\gamma}_{1}, \bar{\gamma}_{2}, \bar{\gamma}_{3}$ obtained from the latter using the expressions in Ref. [46]. The spinorbit splitting energy $\Delta_{\text {so }}$ and the parameter $E_{P}$ describing the mixing of the conduction and valence bands, which is of paramount importance for the evaluation of IVBA, are parameterized as a function of $\mathrm{Sn}$ concentration in Ref. [19]. The bowing parameters for calculation of the direct bandgap $E_{g}$ can be found in Ref. [91]. For SiGeSn alloys, the Luttinger parameters for $\mathrm{Si}$ and for $\mathrm{GeSn}$ were linearly interpolated.

Funding. Deutsche Forschungsgemeinschaft (299480227).

Acknowledgment. We thank Dr. Gergoe Letay (Synopsys Switzerland LLC) for fruitful discussions and helpful suggestions regarding simulations via Synopsys' sDevice modeling toolbox.

Disclosures. The authors declare no conflicts of interests.

\section{REFERENCES}

1. F. Xia, L. Sekaric, and Y. Vlasov, "Ultracompact optical buffers on a silicon chip," Nat. Photonics 1, 65-71 (2007).

2. J. Witzens, "High-speed silicon photonics modulators," Proc. IEEE 106, 2158-2182 (2018).

3. G. Masini, S. Sahni, G. Capellini, J. Witzens, and C. Gunn, "Highspeed near infrared optical receivers based on Ge waveguide photodetectors integrated in a CMOS process," Adv. Opt. Technol. 2008, 196572 (2008).

4. A. Narasimha, S. Abdalla, C. Bradbury, A. Clark, J. Clymore, J. Coyne, A. Dahl, S. Gloekner, A. Gruenberg, D. Guckenberger, S. Gutierrez, M. Harrison, D. Kucharski, K. Leap, R. LeBlanc, Y. Liang, M. Mack, D. Martinez, G. Masini, A. Mekis, R. Menigoz, C. Ogden, M. Peterson, T.
Pinguet, J. Redman, J. Rodriguez, S. Sahni, M. Sharp, T. J. Sleboda, D. Song, Y. Wang, B. Welch, J. Witzens, W. Xu, K. Yokoyama, and P. De Dobbelaere, "An ultra low power CMOS photonics technology platform for $\mathrm{H} / \mathrm{S}$ optoelectronic transceivers at less than $\$ 1$ per Gbps," in Conference on Optical Fiber Communication (OFC) (2010), paper OMV.4.

5. H. Yu, P. Doussiere, D. Patel, W. Lin, K. Al-hemyari, J. Park, C. Jan, R. Herrick, I. Hoshino, L. Busselle, M. Bresnehan, A. Bowles, G. A. Ghiurcan, H. Frish, S. Yerkes, R. Venables, P. Seddighian, X. Serey, K. Nguyen, A. Banerjee, S. Amiralizadeh Asl, Q. Zhu, S. Gupta, A. Fuerst, A. Dahal, J. Chen, Y. Malinge, H. Mahalingam, M. Kwon, S. Gupta, A. Agrawal, R. Narayan, M. Favaro, D. Zhu, and Y. Akulova, "400 Gbps fully integrated DR4 silicon photonics transmitter for data center applications," in Conference on Optical Fiber Communication (OFC) (2020), paper T3H.6.

6. Y. Wan, D. Jung, J. Norman, C. Shang, I. MacFarlane, Q. Li, M. J. Kennedy, A. C. Gossard, K. M. Lau, and J. E. Bowers, "O-band electrically injected quantum dot micro-ring lasers on on-axis (001) GaP/Si and V-groove Si," Opt. Express 25, 26853-26860 (2017).

7. Y. Wang, S. Chen, Y. Yu, L. Zhou, L. Liu, C. Yang, M. Liao, M. Tang, Z. Liu, J. Wu, W. Li, I. Ross, A. J. Seeds, H. Liu, and S. Yu, "Monolithic quantum-dot distributed feedback laser array on silicon," Optica 5, 528-533 (2018).

8. Y. Han, Z. Yan, W. K. Ng, Y. Xue, K. S. Wong, and K. M. Lau, "Bufferless $1.5 \mu \mathrm{m}$ III-V lasers grown on Si-photonics $220 \mathrm{~nm}$ silicon-on-insulator platforms," Optica 7, 148-153 (2020).

9. Y. Shi, B. Kunert, Y. de Koninck, M. Pantouvaki, J. van Campenhout, and D. van Thourhout, "Novel adiabatic coupler for III-V nano-ridge laser grown on a Si photonics platform," Opt. Express 27, 3778137794 (2019).

10. V. Deshpande, H. Hahn, E. O'Connor, Y. Baumgartner, M. Sousa, D. Caimi, H. Boutry, J. Widiez, L. Brevard, C. Le Royer, M. Vinet, J. Fompeyrine, and L. Czornomaz, "First demonstration of 3D SRAM through 3D monolithic integration of InGaAs n-FinFETs on FDSOI Si CMOS with inter-layer contacts," in Symposium on VLSI Technology (IEEE) (2017), pp. T74-T75.

11. J. Liu, X. Sun, R. Camacho-Aguilera, L. C. Kimerling, and J. Michel, "Ge-on-Si laser operating at room temperature," Opt. Lett. 35, 679681 (2010).

12. R. E. Camacho-Aguilera, Y. Cai, N. Patel, J. T. Bessette, M. Romagnoli, L. C. Kimerling, and J. Michel, "An electrically pumped germanium laser," Opt. Express 20, 11316-11320 (2012).

13. R. E. Camacho-Aguilera, "Ge-on-Si LASER for silicon photonics," Ph.D. thesis (Department of Materials Science and Engineering, Massachusetts Institute for Technology, 2013).

14. G. He and H. A. Atwater, "Interband transitions in $\mathrm{Sn}_{x} \mathrm{Ge}_{1-x}$ alloys," Phys. Rev. Lett. 79, 1937-1940 (1997).

15. R. Kitamura, L. Pilon, and M. Jonasz, "Optical constants of silica glass from extreme ultraviolet to far infrared at near room temperature," Appl. Opt. 46, 8118-8133 (2007).

16. R. Soref, "The past, present, and future of silicon photonics," IEEE J. Sel. Top. Quantum Electron. 12, 1678-1687 (2006).

17. R. Soref, "Mid-infrared photonics in silicon and germanium," Nat. Photonics 4, 495-497 (2010).

18. S. A. Ghetmiri, W. Du, J. Margetis, A. Mosleh, L. Cousar, B. R. Conley, L. Domulevicz, A. Nazzal, G. Sun, R. A. Soref, J. Tolle, B. Li, H. A. Naseem, and S.-Q. Yu, "Direct-bandgap GeSn grown on silicon with $2230 \mathrm{~nm}$ photoluminescence," Appl. Phys. Lett. 105, 151109 (2014).

19. S. Wirths, R. Geiger, N. von den Driesch, G. Mussler, T. Stoica, S. Mantl, Z. Ikonic, M. Luysberg, S. Chiussi, J. M. Hartmann, H. Sigg, J. Faist, D. Buca, and D. Grützmacher, "Lasing in direct-bandgap GeSn alloy grown on Si," Nat. Photonics 9, 88-92 (2015).

20. D. Stange, S. Wirths, R. Geiger, C. Schulte-Braucks, B. Marzban, N. von den Driesch, G. Mussler, T. Zabel, T. Stoica, J.-M. Hartmann, S. Mantl, Z. Ikonic, D. Grützmacher, H. Sigg, J. Witzens, and D. Buca, "Optically pumped GeSn microdisk lasers on Si," ACS Photon. $\mathbf{3}$, 1279-1285 (2016).

21. S. Al-Kabi, S. A. Ghetmiri, J. Margetis, T. Pham, Y. Zhou, W. Dou, B. Collier, R. Quinde, W. Du, A. Mosleh, J. Liu, G. Sun, R. A. Soref, J. Tolle, B. Li, M. Mortazavi, H. A. Naseem, and S.-Q. Yu, "An optically 
pumped $2.5 \mu \mathrm{m}$ GeSn laser on Si operating at $110 \mathrm{~K}$," Appl. Phys. Lett. 109, 171105 (2016).

22. J. Margetis, S. Al-Kabi, W. Du, W. Dou, Y. Zhou, T. Pham, P. Grant, S. Ghetmiri, A. Mosleh, B. Li, J. Liu, G. Sun, R. Soref, J. Tolle, M. Mortazavi, and S.-Q. Yu, "Si-based GeSn lasers with wavelength coverage of 2-3 $\mu \mathrm{m}$ and operating temperatures up to $180 \mathrm{~K}$," ACS Photon. 5, 827-833 (2018).

23. Q. M. Thai, N. Pauc, J. Aubin, M. Bertrand, J. Chrétien, V. Delaye, A. Chelnokov, J.-M. Hartmann, V. Reboud, and V. Calvo, "GeSn heterostructure micro-disk laser operating at $230 \mathrm{~K}$," Opt. Express 26. 32500-32508 (2018).

24. Y. Zhou, W. Dou, W. Du, S. Ojo, H. Tran, S. A. Ghetmiri, J. Liu, G. Sun, R. Soref, J. Margetis, J. Tolle, B. Li, Z. Chen, M. Mortazavi, and S.-Q. $\mathrm{Yu}$, "Optically pumped GeSn lasers operating at $270 \mathrm{~K}$ with broad waveguide structures on Si," ACS Photon. 6, 1434-1441 (2019).

25. J. Chrétien, N. Pauc, F. A. Pilon, M. Bertrand, Q.-M. Thai, L. Casiez, N. Bernier, H. Dansas, P. Gergaud, E. Delamadeleine, R. Khazaka, H. Sigg, J. Faist, A. Chelnokov, V. Reboud, J.-M. Hartmann, and V. Calvo, "GeSn lasers covering a wide wavelength range thanks to uniaxial tensile strain," ACS Photon. 6, 2462-2469 (2019).

26. Z. Alferov, "Double heterostructure lasers: early days and future perspectives," IEEE J. Sel. Top. Quantum Electron. 6, 832-840 (2000).

27. R. Chen, S. Gupta, Y.-C. Huang, Y. Huo, C. W. Rudy, E. Sanchez, Y. Kim, T. I. Kamins, K. C. Saraswat, and J. S. Harris, "Demonstration of a $\mathrm{Ge} / \mathrm{GeSn} / \mathrm{Ge}$ quantum-well microdisk resonator on silicon: enabling high-quality $\mathrm{Ge}(\mathrm{Sn})$ materials for micro- and nanophotonics," Nano Lett. 14, 37-43 (2014).

28. D. Stange, N. von den Driesch, D. Rainko, S. Roesgaard, I. Povstugar, J.-M. Hartmann, T. Stoica, Z. Ikonic, S. Mantl, D. Grützmacher, and D. Buca, "Short-wave infrared LEDs from GeSn/ SiGeSn multiple quantum wells," Optica 4, 185-188 (2017).

29. D. Stange, N. von den Driesch, T. Zabel, F. Armand-Pilon, D. Rainko, B. Marzban, P. Zaumseil, J.-M. Hartmann, Z. Ikonic, G. Capellini, S. Mantl, H. Sigg, J. Witzens, D. Grützmacher, and D. Buca, "GeSn/ SiGeSn heterostructure and multi quantum well lasers," ACS Photon. 5, 4628-4636 (2018).

30. N. von den Driesch, D. Stange, D. Rainko, I. Povstugar, P. Zaumseil, G. Capellini, T. Schröder, T. Denneulin, Z. Ikonic, J.-M. Hartmann, H. Sigg, S. Mantl, D. Grützmacher, and D. Buca, "Advanced GeSn/ SiGeSn group IV heterostructure lasers," Adv. Sci. 5, 1700955 (2018).

31. S. Wirths, D. Buca, Z. Ikonic, P. Harrison, A. T. Tiedemann, B. Holländer, T. Stoica, G. Mussler, U. Breuer, and J. M. Hartmann, "SiGeSn growth studies using reduced pressure chemical vapor deposition towards optoelectronic applications," Thin Solid Films 557, 183-187 (2014).

32. A. Elbaz, D. Buca, N. von den Driesch, K. Pantzas, G. Patriarche, N. Zerounian, E. Herth, X. Checoury, S. Sauvage, I. Sagnes, A. Foti, R. Ossikovski, J.-M. Hartmann, F. Boeuf, Z. Ikonic, P. Boucaud, D. Grützmacher, and M. El Kurdi, "Ultra-low-threshold continuous-wave and pulsed lasing in tensile-strained GeSn alloys," Nat. Photonics 14, 375-382 (2020).

33. Y. Zhou, Y. Miao, S. Ojo, H. Tran, G. Abernathy, J. M. Grant, S. Amoah, G. Salamo, W. Du, J. Liu, J. Margetis, J. Tolle, Y.-H. Zhang, G. Sun, R. A. Soref, B. Li, and S.-Q. Yu, "Electrically injected GeSn lasers on Si operating up to 100 K," Optica 7, 924-928 (2020).

34. B. Marzban, J. Nojic, D. Stange, D. Buca, and J. Witzens, "Design of a waveguide-coupled GeSn disk laser," in IEEE Photonics Society Summer Topicals (2020), pp. 1-2.

35. G. Sun, R. A. Soref, and H. H. Cheng, "Design of an electrically pumped $\mathrm{SiGeSn} / \mathrm{GeSn} / \mathrm{SiGeSn}$ double-heterostructure midinfrared laser," J. Appl. Phys. 108, 033107 (2010).

36. G. Sun, R. A. Soref, and H. H. Cheng, "Design of a Si-based latticematched room-temperature GeSn/GeSiSn multi-quantum-well mid-infrared laser diode," Opt. Express 18, 19957-19965 (2010).

37. D. Rainko, Z. Ikonic, N. Vukmirović, D. Stange, N. von den Driesch, D. Grützmacher, and D. Buca, "Investigation of carrier confinement in direct bandgap GeSn/SiGeSn 2D and OD heterostructures," Sci. Rep. 8, 15557 (2018).

38. S. Cho, R. Chen, S. Koo, G. Shambat, H. Lin, N. Park, J. Vuckovic, T. I. Kamins, B.-G. Park, and J. S. Harris, "Fabrication and analysis of epitaxially grown $\mathrm{Ge}_{1-x} \mathrm{Sn}_{x}$ microdisk resonator with 20-nm freespectral range," IEEE Photon. Technol. Lett. 23, 1535-1537 (2011).

39. H.-G. Park, S.-H. Kim, S.-H. Kwon, Y.-G. Ju, J.-K. Yang, J.-H. Baek, S.-B. Kim, and Y.-H. Lee, "Electrically driven single-cell photonic crystal laser," Science 305, 1444-1447 (2004).

40. O. Moutanabbir, S. Assali, X. Gong, E. O'Reilly, C. A. Broderick, B. Marzban, J. Witzens, W. Du, S.-Q. Yu, A. Chelnokov, D. Buca, and D. Nam, "Monolithic infrared silicon photonics: the rise of $(\mathrm{Si})$ GeSn semiconductors," Appl. Phys. Lett. 118, 110502 (2021).

41. R. Geiger, "Direct band gap germanium for Si-compatible lasing," Ph.D. thesis (ETH Zürich, 2016).

42. B. Julsgaard, N. von den Driesch, P. Tidemand-Lichtenberg, C. Pedersen, Z. Ikonic, and D. Buca, "Carrier lifetime of GeSn measured by spectrally resolved picosecond photoluminescence spectroscopy," Photon. Res. 8, 788-798 (2020).

43. S. N. Khatami and Z. Aksamija, "Lattice thermal conductivity of the binary and ternary group-IV alloys Si-Sn, Ge-Sn, and Si-Ge-Sn," Phys. Rev. Appl. 6, 014015 (2016).

44. U. Piesbergen, "The mean atomic heats of the III-V semiconductors: AlSb, GaAs, InP, GaSb, InAs, InSb and the atomic heats of the element Ge between 12 and 273 K," Z. Naturforsch. 18, 141-147 (1963).

45. H. Tran, W. Du, S. A. Ghetmiri, A. Mosleh, G. Sun, R. A. Soref, J. Margetis, J. Tolle, B. Li, H. A. Naseem, and S.-Q. Yu, "Systematic study of $\mathrm{Ge}_{1-x} \mathrm{Sn}_{x}$ absorption coefficient and refractive index for the device applications of Si-based optoelectronics," J. Appl. Phys. 119, 103106 (2016).

46. T. B. Bahder, "Eight-band k.p model of strained zinc-blende crystals," Phys. Rev. B 41, 11992-12001 (1990).

47. K. L. Low, Y. Yang, G. Han, W. Fan, and Y.-C. Yeo, "Electronic band structure and effective mass parameters of $\mathrm{Ge}_{1-x} \mathrm{Sn}_{x}$ alloys," J. Appl. Phys. 112, 103715 (2012).

48. M. Jaros, "Simple analytic model for heterojunction band offsets," Phys. Rev. B 37, 7112-7114 (1988).

49. I. Synopsys, Sentaurus ${ }^{\mathrm{TM}}$ Device User Guide, Version N-2017.09 (Synopsys, 2017)

50. C. R. Crowell, "The Richardson constant for thermionic emission in Schottky barrier diodes," Solid-State Electron. 8, 395-399 (1965).

51. D. Tammaro, K. Hess, and F. Capasso, " $\Gamma-X$ phonon-assisted thermionic currents in the $\mathrm{GaAs} / \mathrm{Al}_{x} \mathrm{Ga}_{1-x}$ As interface system," J. Appl. Phys. 73, 8536-8543 (1993).

52. N. K. Dutta and R. J. Nelson, "The case for Auger recombination in $\mathrm{In}_{1-x} \mathrm{Ga}_{x} A s_{y} \mathrm{P}_{1-y}$, " J. Appl. Phys. 53, 74-92 (1982).

53. N. F. Massé, A. R. Adams, and S. J. Sweeney, "Experimental determination of the band gap dependence of Auger recombination in InGaAs/InP multiple quantum well lasers at room temperature," Appl. Phys. Lett. 90, 161113 (2007).

54. R. Geiger, T. Zabel, and H. Sigg, "Group IV direct band gap photonics: methods, challenges, and opportunities," Front. Mater. 2, 52 (2015).

55. A. Sugimura, "Band-to-band Auger recombination effect on InGaAsP laser threshold," IEEE J. Quantum Electron. 17, 627-635 (1981).

56. W. W. Lui, T. Yamanaka, Y. Yoshikuni, K. Yokoyama, and S. Seki, "Suppression of Auger recombination effects in compressively strained quantum-well lasers," IEEE J. Quantum Electron. 29, 1544-1552 (1993).

57. S. Assali, M. Elsayed, J. Nicolas, M. O. Liedke, A. Wagner, M. Butterling, R. Krause-Rehberg, and O. Moutanabbir, "Vacancy complexes in nonequilibrium germanium-tin semiconductors," Appl. Phys. Lett. 114, 251907 (2019).

58. E. Gaubas and J. Vanhellemont, "Dependence of carrier lifetime in germanium on resisitivity and carrier injection level," Appl. Phys. Lett. 89, 142106 (2006).

59. S. Gupta, R. Chen, J. S. Harris, and K. C. Saraswat, "Atomic layer deposition of $\mathrm{Al}_{2} \mathrm{O}_{3}$ on germanium-tin (GeSn) and impact of wet chemical surface pre-treatment," Appl. Phys. Lett. 103, 241601 (2013).

60. S. Park, K. Yamada, T. Tsuchizawa, T. Watanabe, H. Shinojima, H. Nishi, R. Kou, and S.-I. Itabashi, "Influence of carrier lifetime on performance of silicon $p-i-n$ variable optical attenuators fabricated on submicrometer rib waveguides," Opt. Express 18, 11282-11291 (2010). 
61. N. von den Driesch, "Epitaxy of group IV Si-Ge-Sn alloys for advanced heterostructure light emitters," Ph.D. thesis (Faculty of Mathematics, Computer Science and Natural Sciences, RWTH Aachen, 2017).

62. C. Schulte-Braucks, "Investigation of GeSn as novel group IV semiconductor for electronic applications," Ph.D. thesis (Faculty of Mathematics, Computer Science and Natural Sciences, RWTH Aachen, 2017).

63. O. A. Golikova, B. Y. Moizhes, and L. S. Stilbans, "Hole mobility of germanium as a function of concentration and temperature," Sov. Phys. Solid State 3, 2259-2265 (1962).

64. N. D. Arora, J. R. Hauser, and D. J. Roulston, "Electron and hole mobilities in silicon as a function of concentration and temperature," IEEE Trans. Electron. Devices 29, 292-295 (1982).

65. Supplementary Information available at http://www.doi.org/10.6084/ m9.figshare.14115407.

66. L. A. Coldren, S. W. Corzine, and M. Mashanovitch, Diode Lasers and Photonic Integrated Circuits, 2nd ed. (Wiley, 2012), Appx. 8.

67. L. A. Coldren, S. W. Corzine, and M. Mashanovitch, Diode Lasers and Photonic Integrated Circuits, 2nd ed. (Wiley, 2012), Chap. 4.

68. D. V. Karasyov and V. K. Kononenko, "Broadening of gain spectra of quantum-well lasers," J. Appl. Spectrosc. 61, 739-743 (1994).

69. R. Nagarajan, T. Kamiya, and A. Kurobe, "Band filling in GaAs/ AIGaAs multiquantum well lasers and its effect on the threshold current," IEEE J. Quantum Electron. 25, 1161-1170 (1989).

70. A. A. Afonenko, V. K. Kononenko, and A. Joullié, "On spectral line broadening in quantum-well heterostructure lasers," Proc. SPIE 6184, 618428 (2006).

71. M. Prost, M. El Kurdi, F. Aniel, N. Zerounian, S. Sauvage, X. Checoury, F. Bœuf, and P. Boucaud, "Analysis of optical gain threshold in n-doped and tensile-strained germanium heterostructure diodes," J. Appl. Phys. 118, 125704 (2015).

72. J. I. Pankove and P. Aigrain, "Optical absorption of arsenic-doped degenerate germanium," Phys. Rev. 126, 956-962 (1962).

73. R. Camacho-Aguilera, Z. Han, Y. Cai, L. C. Kimerling, and J. Michel, "Direct band gap narrowing in highly doped Ge," Appl. Phys. Lett. 102, 152106 (2013).

74. S. C. Jain and D. J. Roulston, "A simple expression for band gap narrowing (BGN) in heavily doped $\mathrm{Si}, \mathrm{Ge}, \mathrm{GaAs}$ and $\mathrm{Ge}_{x} \mathrm{Si}_{1-x}$ strained layers," Solid State Electron. 34, 453-465 (1991).

75. J. T. Robinson, K. Preston, O. Painter, and M. Lipson, "First-principle derivation of gain in high-index-contrast waveguides," Opt. Express 16, 16659-16669 (2008).

76. M. Heiblum and J. Harris, "Analysis of curved optical waveguides by conformal transformation," IEEE J. Quantum Electron. 11, 75-83 (1975).

77. R. Soref and B. Bennett, "Electrooptical effects in silicon," IEEE J. Quantum Electron. 23, 123-129 (1987).
78. A. Afzali-Kushaa and G. I. Haddad, "Effects of biaxial strain on the intervalence-band absorption spectra of InGaAs/InP systems," J. Appl. Phys. 77, 6549-6556 (1995).

79. C.-Y. Tsai, C.-Y. Tsai, C.-H. Chen, T.-L. Sung, T.-Y. Wu, and F.-P. Shih, "Theoretical model for intravalley and intervalley free-carrier absorption in semiconductor lasers: beyond the classical Drude model," IEEE J. Quantum Electron. 34, 552-559 (1998).

80. M. Nedeljkovic, R. Soref, and G. Z. Mashanovich, "Predictions of freecarrier electroabsorption and electrorefraction in germanium," IEEE Photon. J. 7, 2600214 (2015).

81. R. Newman and W. W. Tyler, "Effect of impurities on free-hole infrared absorption in p-type germanium," Phys. Rev. 105, 885-886 (1957).

82. E. Jaberansary, T. M. B. Masaud, M. M. Milosevic, M. Nedeljkovic, G. Z. Mashanovich, and H. M. H. Chong, "Scattering loss estimation using 2-D Fourier analysis and modeling of sidewall roughness on optical waveguides," IEEE Photon. J. 5, 6601010 (2013).

83. V. R. D'Costa, C. S. Cook, A. G. Birdwell, C. L. Littler, M. Canonico, S. Zollner, J. Kouvetakis, and J. Menéndez, "Optical critical points of thinfilm $\mathrm{Ge}_{1-y} \mathrm{Sn}_{y}$ alloys: a comparative $\mathrm{Ge}_{1-y} \mathrm{Sn}_{y} / \mathrm{Ge}_{1-x} \mathrm{Si}_{x}$ study," Phys. Rev. B 73, 125207 (2006).

84. W.-J. Yin, X.-G. Gong, and S.-H. Wei, "Origin of the unusually large band-gap bowing and the breakdown of the band-edge distribution rule in the $\mathrm{Sn}_{x} \mathrm{Ge}_{1-x}$ alloys," Phys. Rev. B 78, 161203 (2008).

85. A. Kurobe, H. Furuyama, S. Naritsuka, N. Sugiyama, Y. Kokubun, and M. Nakamura, "Effects of well number, cavity length, and facet reflectivity on the reduction of threshold current of GaAs/AIGaAs multiquantum well lasers," IEEE J. Quantum Electron. 24, 635-640 (1988).

86. P. C. Grant, J. Margetis, W. Du, Y. Zhou, W. Dou, G. Abernathy, A. Kuchuk, B. Li, J. Tolle, J. Liu, G. Sun, R. A. Soref, M. Mortazavi, and S.-Q. Yu, "Study of direct bandgap type-I GeSn/GeSn double quantum well with improved carrier confinement," Nanotechnology 29, 465201 (2018).

87. S. Assali, A. Dijkstra, A. Attiaoui, É. Bouthillier, J. E. M. Haverkort, and O. Moutanabbir, "Midinfrared emission and absorption in strained and relaxed direct band-Gap $\mathrm{Ge}_{1-x} \mathrm{Sn}_{x}$ semiconductors," Phys. Rev. Appl. 15, 024031 (2021).

88. Y. C. Chang and R. B. James, "Saturation of intersubband transitions in p-type semiconductor quantum wells," Phys. Rev. B 39, 1267212681 (1989).

89. S. L. Chuang, "Chapter 4: theory of electronic band structures in semiconductors," in Physics of Optoelectronic Devices (Wiley, 1995).

90. Y.-M. Mu and S. S. Pei, "Effects of anisotropic k.p interactions on energy bands and optical properties of type-II interband cascade lasers," J. Appl. Phys. 96, 1866-1879 (2004).

91. M.-Y. Ryu, T. R. Harris, Y. K. Yeo, R. T. Beeler, and J. Kouvetakis, "Temperature-dependent photoluminescence of $\mathrm{Ge} / \mathrm{Si}$ and $\mathrm{Ge}_{1-y} \mathrm{Sn}_{y} /$ $\mathrm{Si}$, indicating possible indirect-to-direct bandgap transition at lower Sn content," Appl. Phys. Lett. 102, 171908 (2013). 